\title{
Translocation of CaMKII to dendritic microtubules supports the plasticity of local synapses
}

\author{
Mado Lemieux, ${ }^{1}$ Simon Labrecque, ${ }^{1}$ Christian Tardif, Étienne Labrie-Dion, ${ }^{1}$ Éric LeBel, ${ }^{1}$ and Paul De Koninck ${ }^{1,2}$ \\ 'Institut universitaire en santé mentale de Québec, Québec G1J 2G3, Canada \\ 2Département de Biochimie, Microbiologie et Bio-informatique, Université Laval, Québec GIV OA6, Canada
}

$\mathrm{T}$

he processing of excitatory synaptic inputs involves compartmentalized dendritic $\mathrm{Ca}^{2+}$ oscillations. The downstream signaling evoked by these local $\mathrm{Ca}^{2+}$ transients and their impact on local synaptic development and remodeling are unknown. $\mathrm{Ca}^{2+} /$ calmodulindependent protein kinase II (CaMKII) is an important decoder of $\mathrm{Ca}^{2+}$ signals and mediator of synaptic plasticity. In addition to its known accumulation at spines, we observed with live imaging the dynamic recruitment of CaMKII to dendritic subdomains adjacent to activated synapses in cultured hippocampal neurons. This localized and transient enrichment of CaMKII to dendritic sites coincided spatially and temporally with dendritic $\mathrm{Ca}^{2+}$ transients. We show that it involved an interaction with microtubular elements, required activation of the kinase, and led to localized dendritic CaMKII autophosphorylation. This process was accompanied by the adjacent remodeling of spines and synaptic AMPA receptor insertion. Replacement of endogenous CaMKIl with a mutant that cannot translocate within dendrites lessened this activity-dependent synaptic plasticity. Thus, CaMKII could decode compartmental dendritic $\mathrm{Ca}^{2+}$ transients to support remodeling of local synapses.

\section{Introduction}

Input-specific synaptic plasticity is thought to involve an activitydependent and synapse-specific recruitment of proteins that affect synaptic strength. The "synaptic tag and capture" hypothesis proposed by Morris and colleagues (Frey and Morris, 1998; Redondo and Morris, 2011) postulates that modifications in the activated synapse lead to a tag that can then capture specific material among all of which is delivered throughout the dendritic arbors. However, to ensure appropriate delivery of material to activated synapses, a missing mechanism is one to guide such material from the dendrite into those synapses. How could this be achieved? One possibility would involve a signal, arising at the base of active spines within the dendritic shaft, which would impact on the local production and/or release of material for its subsequent translocation into active spines for capture.

Activity-dependent dendritic signals known to occur near active synapses are $\mathrm{Ca}^{2+}$ spikes (Augustine et al., 2003), and an emerging concept in dendritic processing is the compartmentalization of the $\mathrm{Ca}^{2+}$ events, possibly linked to clustered localized inputs (Chalifoux and Carter, 2011; Kleindienst et al., 2011;

Correspondence to Paul De Koninck: paul.dekoninck@neurosciences.ulaval.ca Abbreviations used in this paper: AMPAR, $\alpha$-amino-3-hydroxy-5-methyl4-isoxazolepropionic acid receptor; CaM, calmodulin; CaMKII, $\mathrm{Ca}^{2+} /$ calmodulindependent protein kinase II; cLTP, long-term potentiation in culture; Glu, glutamate; Gly, glycine; NMDAR, N-methyl-D-aspartate receptor; WT, wild type.
Takahashi et al., 2012). It has been proposed that the preferred site for long-term memory storage in neurons would reside in dendritic branches and subcompartments (Govindarajan et al., 2006, 2011), but the supporting signal processing inside dendrites is unknown. A major decoder of $\mathrm{Ca}^{2+}$ spikes is CaMKII, a key enzyme in activity-dependent synaptic plasticity (Lisman et al., 2012). An interesting feature of CaMKII, which has been revealed by time-lapse imaging techniques, is that its $\mathrm{Ca}^{2+} / \mathrm{CaM}$ activation can lead to its recruitment to sites where $\mathrm{Ca}^{2+}$ rises, such as within activated synapses (Shen and Meyer, 1999; Colbran, 2004; Hudmon et al., 2005; Bayer et al., 2006). If CaMKII was also recruited to dendritic sites where $\mathrm{Ca}^{2+}$ events occur, it might then engage specific signaling that is required to support the plasticity of neighboring synapses.

However, there is no clear evidence that CaMKII is timely located in relevant dendritic sites to be able to affect the plasticity of activated synapses. Using combinations of fluorescent proteins transfected in cultured hippocampal neurons for dual-color time-lapse optical imaging, we examined the spatial and temporal dynamics of CaMKII with those

(c) 2012 Lemieux et al. This article is distributed under the terms of an AttributionNoncommercial-Share Alike-No Mirror Sites license for the first six months after the publication date (see http://www.rupress.org/terms). After six months it is available under a Creative Commons License (Attribution-Noncommercial-Share Alike 3.0 Unported license, as described at http://creativecommons.org/licenses/by-nc-sa/3.0/). 
of $\mathrm{Ca}^{2+}, \alpha$-amino-3-hydroxy-5-methyl-4-isoxazolepropionic acid receptors (AMPARs), and spines during protocols that induce synaptic activity and remodeling. Our experiments newly describe a dynamic process of localized dendritic translocation of CaMKII that requires local $\mathrm{Ca}^{2+}$ activity and microtubular interactions, and which correlates with localized synaptic plasticity. A role for CaMKII dendritic translocation in supporting synaptic plasticity is further corroborated by replacing CaMKII with a mutant that cannot translocate specifically to dendritic domains. Our experiments suggest that CaMKII has a role in dendritic processing, impacting on localized synaptic remodeling.

\section{Results}

Synaptic stimulation leads to the local accumulation of CaMKII to dendritic domains

Several studies have shown that CaMKII translocates to postsynaptic sites after the application of glutamate or NMDA in cultured neurons (Shen and Meyer, 1999; Bayer et al., 2001, 2006; Otmakhov et al., 2004; Hudmon et al., 2005). This translocation requires $\mathrm{Ca}^{2+}$ influx and the $\mathrm{Ca}^{2+} / \mathrm{CaM}$ activation of the enzyme which in turn binds to postsynaptic partners including the $N$-methyl-D-aspartate receptor (NMDAR). Meanwhile, the activation of a subset of excitatory synapses has been shown to increase $\mathrm{Ca}^{2+}$ in dendritic subdomains (Augustine et al., 2003; Kleindienst et al., 2011; Takahashi et al., 2012). We thus wondered whether these dendritic $\mathrm{Ca}^{2+}$ events might recruit CaMKII. We performed time-lapse imaging on cultured neurons that were transfected with mGFP- $\alpha \mathrm{CaMKII}$ and stimulated with a protocol $\left(0 \mathrm{Mg}^{2+} /\right.$ Glycine $)$ known to favor synaptic NMDAR activation and long-term potentiation in culture (cLTP; Lu et al., 2001). Whereas glutamate/glycine (Glu/Gly) stimulation led to the translocation of $\mathrm{mGFP}-\alpha \mathrm{CaMKII}$ to nearly all postsynaptic sites (Fig. S1 A; Hudmon et al., 2005; Bayer et al., 2006), cLTP stimulation led to its translocation to a subset of excitatory synapses (Fig. 1 A; Fig. S1 B), presumably those where sufficient presynaptic glutamate was released. However, we also observed an increase in mGFP- $\alpha$ CaMKII fluorescence immediately adjacent to several of those synapses, as dendritic hot spots (Fig. 1 A; Video 1). The accumulation of mGFP- $\alpha$ CaMKII in dendritic domains generally appeared immediately at the base of activated excitatory synapses, whereas the surrounding domains $(\sim 5 \mu \mathrm{m}$ away from the activated synapse) exhibited a decrease in fluorescence (Fig. 1 B; Fig. S1 B). In cases where neurons responded more strongly to the stimulation by exhibiting synaptic translocation of CaMKII to every spine, we did not observe localized dendritic hot spots (as with bath application of glutamate or NMDA; Fig. S1 A). Spontaneous neuronal activity in standard solution (Fig. S1 C; Video 2) or during increased activity in the presence of $\mathrm{GABA}_{\mathrm{A}}$ receptor blocker bicuculline (Fig. S1 D) also triggered dendritic translocation, but not in activity-blocking solution. We also observed, by immunostaining, the accumulation of endogenous $\alpha \mathrm{CaMKII}$ into local dendritic hot spots (Fig. S2 A).

Dendritic hot spots of mGFP- $\alpha$ CaMKII ranged from $\sim 1-10 \mu \mathrm{m}$ in length and could be observed anywhere throughout the dendritic arbors. We counted from 0 to 20 dendritic translocation sites per neuron analyzed during a 5-min stimulation period (mean $7.4 \pm 1.5 ; n=16$ neurons). Although a fraction of mGFP- $\alpha$ CaMKII that translocated to postsynaptic sites during stimulation was retained upon washout, as described previously (Bayer et al., 2006), the dendritic accumulation of CaMKII was fully reversible (Fig. $1 \mathrm{~B}$; percentage of retention: synapses [32.2 \pm 7.0$]$, dendrites $[1.4 \pm 4.8], \mathrm{P}=0.0024$ two-sided signed rank test; $n=12$ pairs of synaptic/dendritic regions, 6 neurons). Repetitive $0 \mathrm{Mg}^{2+} / \mathrm{Gly}$ stimulations could induce dendritic hot spots of mGFP- $\alpha$ CaMKII, either at the same location or occasionally at new dendritic sites (Video 3). Blocking NMDARs with $50 \mu \mathrm{M}$ D-AP5, $\mathrm{Na}^{2+}$ channels with $1 \mu \mathrm{M}$ TTX, or voltagegated $\mathrm{Ca}^{2+}$ channels with $10 \mu \mathrm{M} \mathrm{Cd}^{2+}$ strongly reduced the accumulation of CaMKII to localized dendritic sites. Blocking $\mathrm{Ca}^{2+}$ release from intracellular stores with $10 \mu \mathrm{M}$ CPA partially reduced $(\sim 40 \%)$ the subdendritic translocation of the kinase (Fig. $1 \mathrm{C}$ ). The effects of these pharmacological blockers indicate that the translocation of CaMKII to subdendritic regions is dependent on the rise of intracellular free $\mathrm{Ca}^{2+}$.

\section{CaMKII translocates to dendritic sites of higher $\mathrm{Ca}^{2+}$ concentration}

To test whether this localized dendritic accumulation of CaMKII spatially and temporally coincides with $\mathrm{Ca}^{2+}$ elevation, we monitored in the same neurons the dynamics of mCherry- $\alpha$ CaMKII and GCaMP2, a genetically encoded $\mathrm{Ca}^{2+}$ indicator (Tallini et al., 2006). At the onset of the $0 \mathrm{Mg}^{2+} / \mathrm{Gly}$ stimulation, $\mathrm{Ca}^{2+}$ transiently increased throughout the neurons, but then hot spots of elevated free $\mathrm{Ca}^{2+}$ oscillations appeared in dendritic segments (Fig. 2 Aii; Video 4). Interestingly, mCherry- $\alpha$ CaMKII gradually accumulated in those same dendritic regions, but not in regions where the elevation in $\mathrm{Ca}^{2+}$ was less pronounced (Fig. $2 \mathrm{~A}$ ). These results indicate that $\mathrm{CaMKII}$ translocates to regions where $\mathrm{Ca}^{2+}$ reaches a significant level during synaptic transmission, which includes active spines and specific dendritic domains.

\section{Confined dendritic translocation of CaMKII} evoked by local glutamate/glycine application To control more directly the localization of $\alpha$ CaMKII translocation inside dendrites, we caused a local elevation of $\mathrm{Ca}^{2+}$ by stimulating a short dendritic segment with Glu/Gly application from a patch pipette placed within a few micrometers of a dendrite. We confirmed that the response was local by monitoring free $\mathrm{Ca}^{2+}$ elevation with GCaMP2, while observing CaMKII translocation with mCherry- $\alpha$ CaMKII (Fig. 2 B). The application of Glu/Gly led to a rapid and localized increase in dendritic $\mathrm{Ca}^{2+}$ near the pipette, which was maintained during a continuous train of stimulating puffs. Meanwhile, a gradual accumulation of mCherry- $\alpha$ CaMKII occurred in the same confined dendritic location (Fig. 2 B).

\section{Dynamic translocation of CaMKII to microtubular elements}

To test whether the local accumulation of CaMKII within dendritic segments arises from its binding to intracellular domains, we performed FRAP measurements (Fig. 3 A) during 

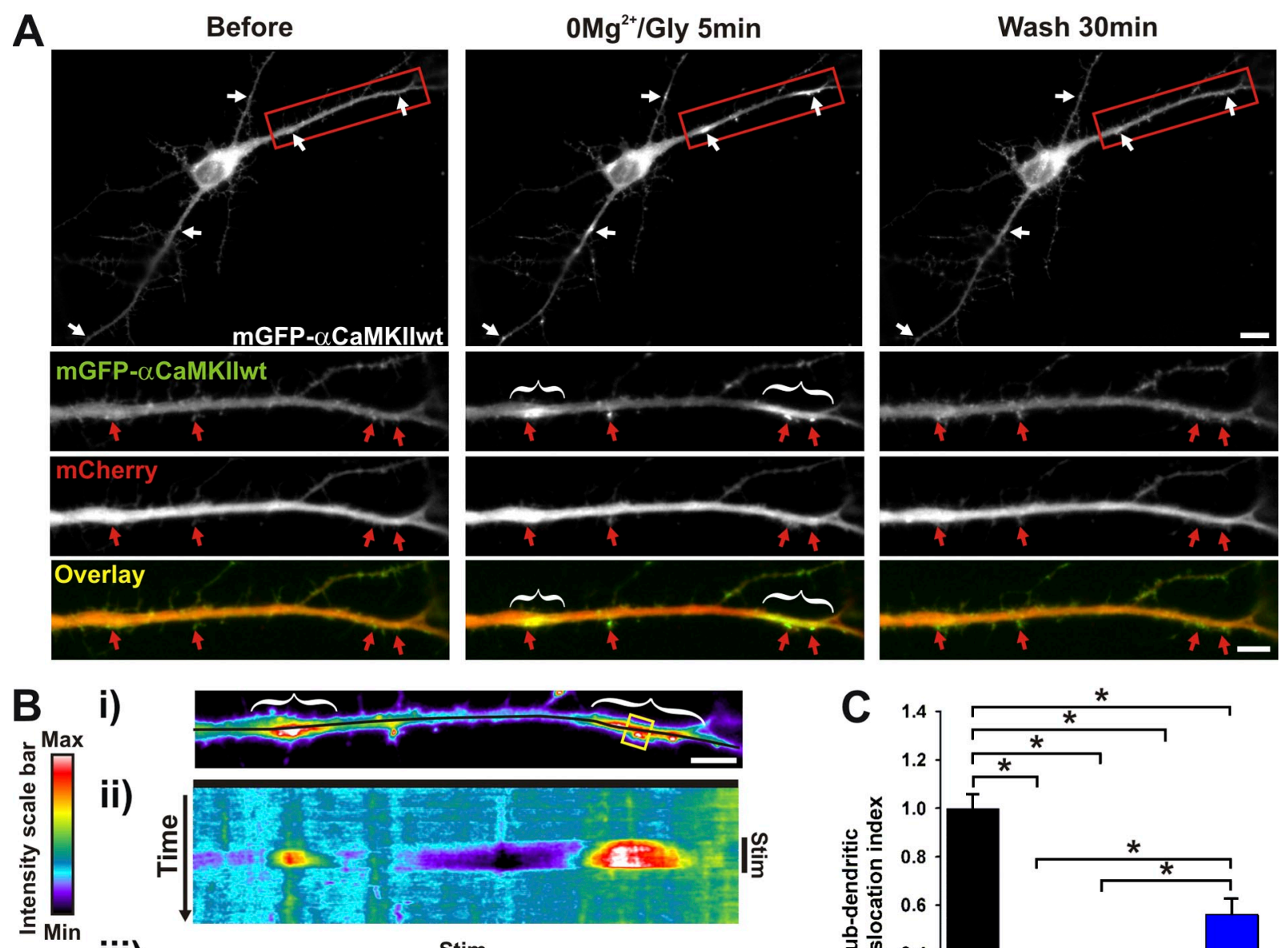

ii)
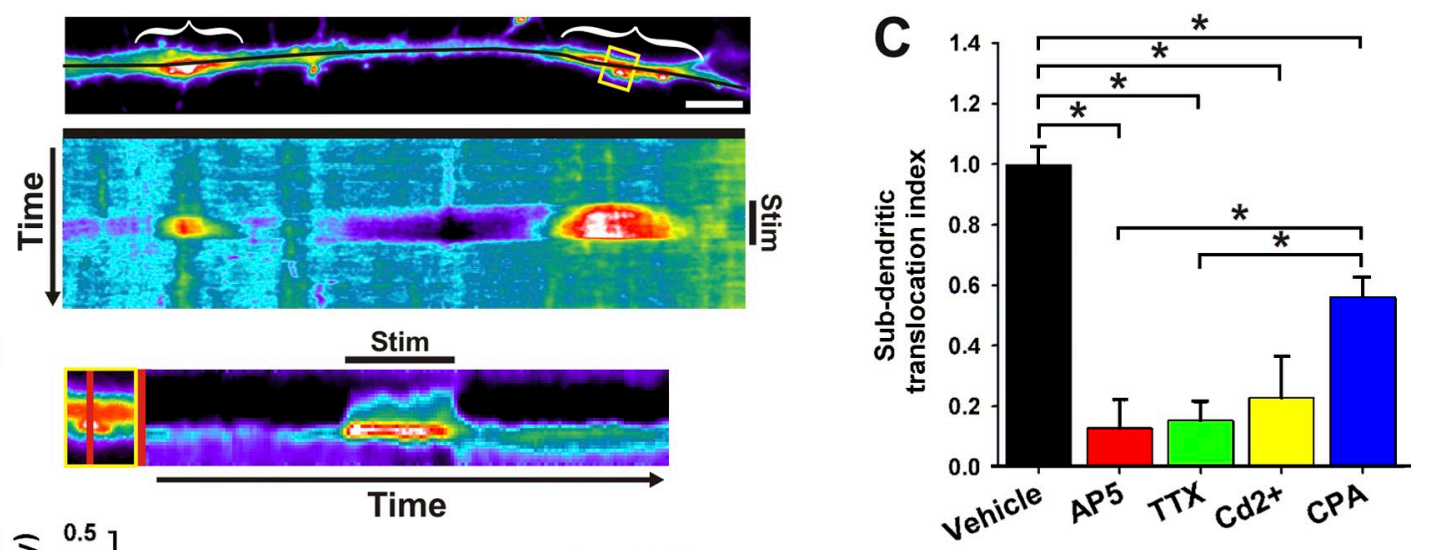

venicle AP5 THX Cd2 CPA

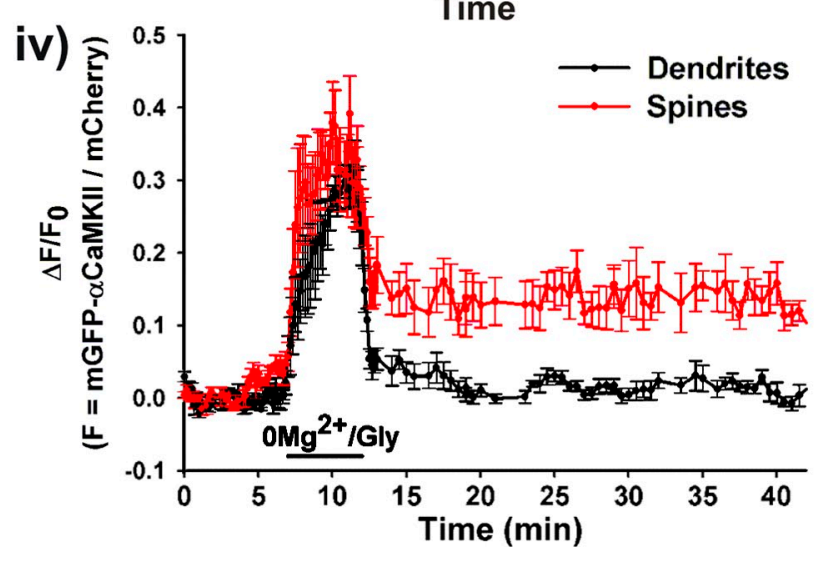

iii)

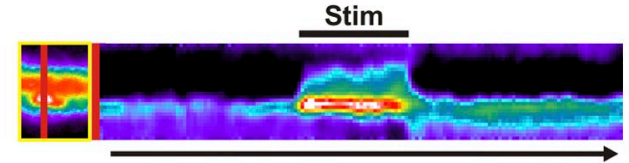

Time

Figure 1. Activity-dependent translocation of $\alpha$ CaMKII to dendritic sites near synapses. (A) Hippocampal neuron (12 DIV) expressing mGFP- $\alpha$ CaMKII and $\mathrm{mCherry}$ imaged before, during, and after stimulation with $0 \mathrm{Mg}^{2+} / \mathrm{Gly}$ for $5 \mathrm{~min}$. (B, i) Pseudocolor image from dendrite in $\mathrm{A}$. Kymograph (ii-iii) and time-lapse (iv) analyses of the change in the fluorescent intensity ratio (mGFP- $\alpha$ CaMKII/mCherry) over time across the dendrite (i-ii, black line) and across the spine (iii, yellow box, red line). Stimulation period is indicated (Stim). (iv) $n=4$ spines and subdendritic regions from the neuron shown in A. Red and white arrows (or brackets) point, respectively, to synaptic and dendritic sites where CaMKII translocated. Bars: (neuron) $10 \mu \mathrm{m}$; (dendrite) $5 \mu \mathrm{m}$. (C) Normalized ratio $( \pm S E M)$ of dendritic segment where $\mathrm{mGFP}-\alpha \mathrm{CaMKII}$ accumulated over total dendritic length after a 5 -min $0 \mathrm{Mg}^{2+} / \mathrm{Gly}$ stimulation $(\mathrm{Vehicle})$, in the presence of $50 \mu \mathrm{M}$ AP5, $1 \mu \mathrm{M}$ TTX, $10 \mu \mathrm{M} \mathrm{Cd}^{2+}$, or $10 \mu \mathrm{M}$ CPA. $n=7-24$ neurons per condition. ${ }^{*}, \mathrm{P}<0.05$ Kruskal-Wallis followed by Tukey's least-significant difference test. See also Fig. S1, Fig. S2, and Videos 1-3.

and after a $0 \mathrm{Mg}^{2+} / \mathrm{Gly}$ stimulation in two regions simultaneously: (i) one at a site of dendritic CaMKII hot spot (D+), but specifically on its edge to avoid bleaching the associated postsynaptic site, and (ii) another a few micrometers away (D-).
During the stimulation, CaMKII was less mobile at the sites where it was concentrated compared with the adjacent region, whereas there was no significant difference between those regions after the stimulation. This result suggests that 

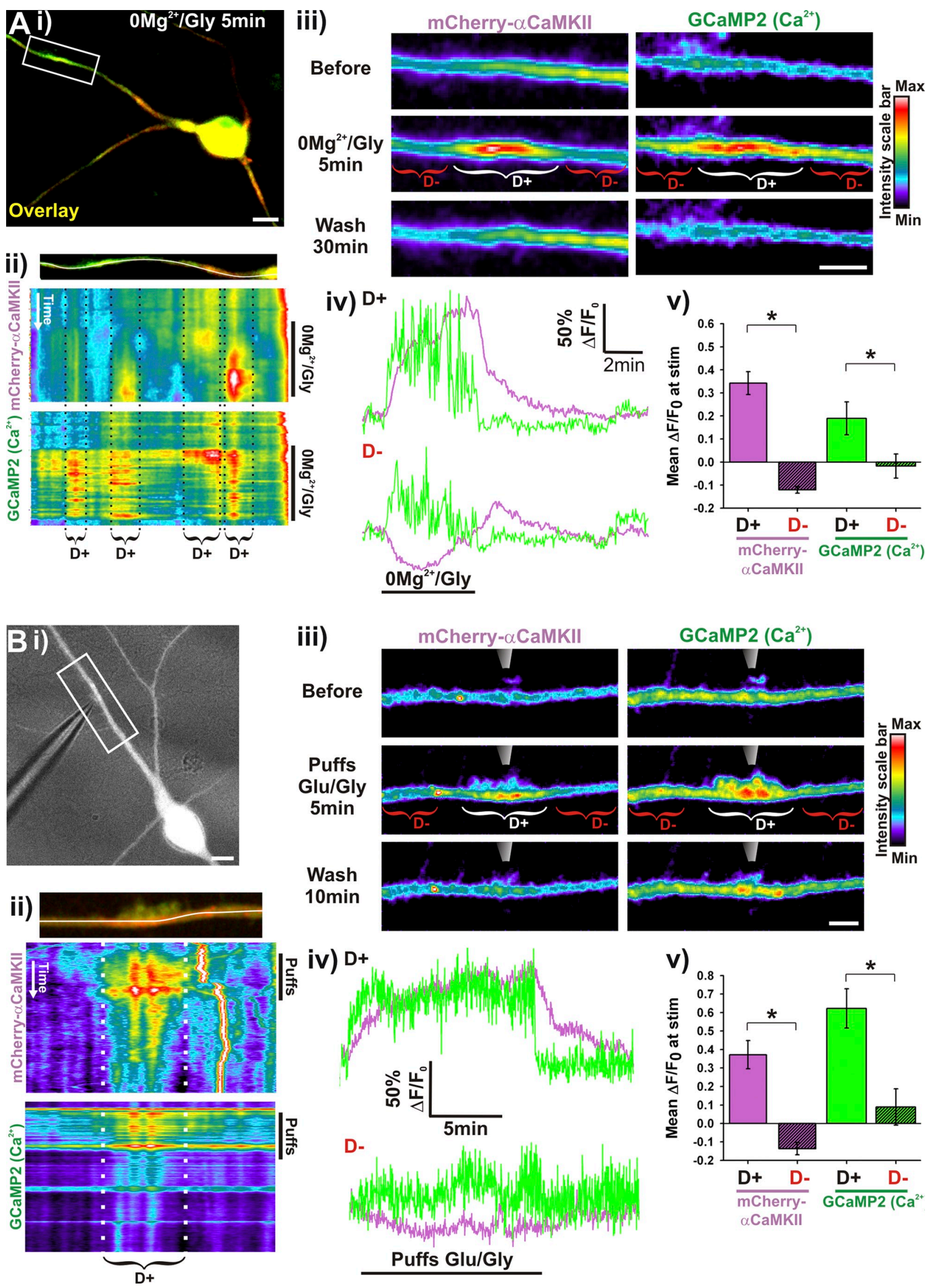

Puffs Glu/Gly 
localized differences in CaMKII binding lead to its accumulation in dendritic domains.

Which dendritic domains could CaMKII bind to? Many years ago, Schulman (1984) identified a microtubule-associated protein (MAP2) as a major substrate of CaMKII. Other MAPs have since then been described as CaMKII substrates (Baudier and Cole, 1987; Baratier et al., 2006). Electron microscopy studies have also revealed CaMKII to be in close proximity with microtubules and neurofilaments (Vallano et al., 1986). Our optical measurements cannot easily resolve microtubules in thin dendrites. However, in larger proximal dendrites, a fiber-like pattern of fluorescence at the base of postsynaptic sites during the $\mathrm{OMg}^{2+} /$ Gly stimulation became apparent (Fig. 3 B; Video 5).

If CaMKII indeed binds to microtubular elements upon $\mathrm{Ca}^{2+}$ elevation, then global stimulation of a neuron should trigger microtubular patterns of mGFP- $\alpha \mathrm{CaMKII}$ fluorescence in the soma, where microtubules are easier to detect. Incubating the cells in a ringer solution that blocks much of the synaptic and electrical activities (high $\mathrm{Mg}^{2+} /$ low $\mathrm{Ca}^{2+}$ solution) revealed a diffuse pattern of fluorescence of $\mathrm{mGFP}-\alpha \mathrm{CaMKII}$ throughout the neurons (Fig. 3 C, top). By contrast, a global stimulation (40 $\mathrm{mM} \mathrm{KCl}$ or Glu/Gly) led to the rapid appearance of fiber patterns of fluorescence clearly visible in the soma and proximal dendrites (Fig. 3 C, middle; Video 6), accompanied by the postsynaptic translocation of the enzyme throughout the dendritic trees. To confirm that CaMKII indeed "decorates" microtubular elements after stimulation, we used immunostaining for endogenous $\alpha$ CaMKII and cytoskeletal markers. The enzyme colocalized strongly with tubulin (Fig. S2, B and D), but less with neurofilaments (NF-M; Fig. S2, C and D). To observe this microtubule decoration dynamically, we also cotransfected neurons with mCherry- $\alpha$ CaMKII and GFP-MAP2B, which revealed a clear increase in overlap during depolarization (Fig. 3 D). Interestingly, the overexpression of GFP-MAP2B by itself increased the level of mCherry-CaMKII localization to microtubular elements, even before stimulation (Fig. $3 \mathrm{~F}$ ). To validate further the interpretation that CaMKII binds to microtubular elements during neuronal activity, we treated neurons with nocodazole for $90 \mathrm{~min}$, to partially destabilize microtubules (Conde and Cáceres, 2009; Fig. S3). Fig. 3 E shows that indeed the partial destabilization of microtubules reduced CaMKII binding in dendrites during stimulation. The remaining fraction of activity-dependent binding occurs potentially on the stable microtubules that resisted nocodazole treatment (Fig. S3). These results argue that the binding to microtubular domains is responsible for the reduced mobility of CaMKII in active dendrites, and that upon neuronal stimulation CaMKII translocates to distinct sites: microtubular elements and postsynaptic sites. $\beta C$ aMKII is known to interact with F-actin filaments, but when neurons are stimulated, $\mathrm{Ca}^{2+} / \mathrm{CaM}$ activation of the enzyme disrupts the interaction (Shen and Meyer, 1999). We imaged mGFP- $\beta$ CaMKII and observed that its fluorescence went from an F-actin-like punctated pattern to a mixture of microtubular and postsynaptic patterns (Fig. S4 C). After synaptic stimulation $\left(\mathrm{OMg}^{2+} / \mathrm{Gly}\right)$, we also observed the confined dendritic accumulation of $\beta \mathrm{CaMKII}$ near activated synapses (Fig. S4, $\mathrm{A}$ and $\mathrm{B}$ ). Thus, the two main subunits of CaMKII in the brain translocate to microtubular domains and postsynaptic sites upon neuronal stimulation.

\section{Mutagenesis analysis of CaMKII}

translocation to microtubular elements

To investigate the mechanisms of CaMKII binding to microtubular domains, we compared the ability of various CaMKII mutants to redistribute to somatodendritic fiber-like regions in response to $\mathrm{KCl}$ stimulation. To minimize the impact of possible mixing of endogenous CaMKII with the mutants, we cotransfected shRNAs for both the $\alpha$ and $\beta$ isoforms, along with shRNA-resistant mGFP-CaMKII mutants. This method reduced the amount of endogenous $\alpha$ - and $\beta$ CaMKII down to $\sim 19 \pm 2 \%$ and $26 \pm 2 \%$, respectively (Fig. S5, B and C). Those shRNA treatments did not impact on the peak $\mathrm{Ca}^{2+}$ response of the neuron during a $\mathrm{KCl}$ stimulation (Fig. $4 \mathrm{~A}$ ), and cotransfected mGFP- $\alpha$ CaMKII wild-type (WT) resistant to shRNA normally exhibited microtubular translocation (Fig. S5 A; Fig. 4 B).

The tested CaMKII mutants were selected on the basis of previous work on synaptic translocation of CaMKII (Bayer et al., 2001, 2006; Hudmon et al., 2005; Marsden et al., 2010) and the mutagenesis analysis by Yang and Schulman (1999). In analyzing the $\mathrm{KCl}$-evoked redistribution of the fluorescence for the different constructs, we can conclude that the activitydependent translocation of CaMKII to microtubular elements shares a number of similarities with that to spines. First, it requires its $\mathrm{Ca}^{2+} / \mathrm{CaM}$ activation, as demonstrated by the lack of microtubular translocation of the mutant (T305-306D) that has impaired binding to $\mathrm{Ca}^{2+} / \mathrm{CaM}$ (Fig. $4 \mathrm{D}$; Rich and Schulman, 1998). Moreover, adding $10 \mu \mathrm{M}$ KN93, a drug preventing CaMKII activation, also blocked its microtubular translocation (but not its inactive analogue KN92; Fig. 4 E). We found that preventing (T286A) or mimicking (T286D) autophosphorylation had little impact on microtubular translocation of the enzyme (Fig. 4, C and D). The complete multimeric structure of the enzyme is required for postsynaptic translocation (Bayer et al., 2006). Likewise, a CaMKII monomer (aa 1-326) or its association domains (aa 315-478) could not translocate to microtubular elements. The kinase domain T-site seems also involved in microtubular binding because the I205K mutation

Figure 2. $\alpha$ CaMKII translocates to dendritic sites of higher $\mathrm{Ca}^{2+}$ concentration. (A) Neurons expressing mCherry- $\alpha \mathrm{CaMKII}$ and GCaMP2 stimulated for 5 min with $\mathrm{OMg}^{2+} / \mathrm{Gly}(\mathrm{Ai}$, iii) or (B) stimulated by local puffs (10-ms puffs at $2 \mathrm{~Hz}$ during $13 \mathrm{~min}$ ) of Glu/Gly (100/10 $\mu \mathrm{M}$; Bi, iii). Brackets indicate CaMKIl dendritic hot spots ( $D+$, white) or adjacent dendritic region (D-, red). (Aii and Bii) Kymograph of the change in intensity of GCaMP2 and mCherry$\alpha$ CaMKII over time across the dendritic region shown above (white line). Stimulation period is indicated $\left(\mathrm{OMg}^{2+} / \mathrm{Gly}\right.$ or Puffs). (Aiv and Biv) Time-lapse analysis of $m$ Cherry- $\alpha$ CaMKII (purple) and GCaMP2 (green) intensity in a region of dendritic CaMKIl translocation (D+, top graph) or in an adjacent region (D-, bottom graph). (Av and Bv) Mean $( \pm S E M) \Delta F / F_{0}$ (in paired regions as in ii-iii) of mCherry- $\alpha$ CaMKIl (purple) and GCaMP2 (green) during the first 5 min of stimulation. (Aiv) $n=12$ neurons, 52 dendritic segments. (Biv) $n=5$ neurons, 5 dendritic segments. Bars: (neurons) $10 \mu \mathrm{m}$; (dendrites) $5 \mu \mathrm{m}$. ${ }^{*}, \mathrm{P}<0.01$ paired $t$ test. See also Video 4 . 


\section{A i)}
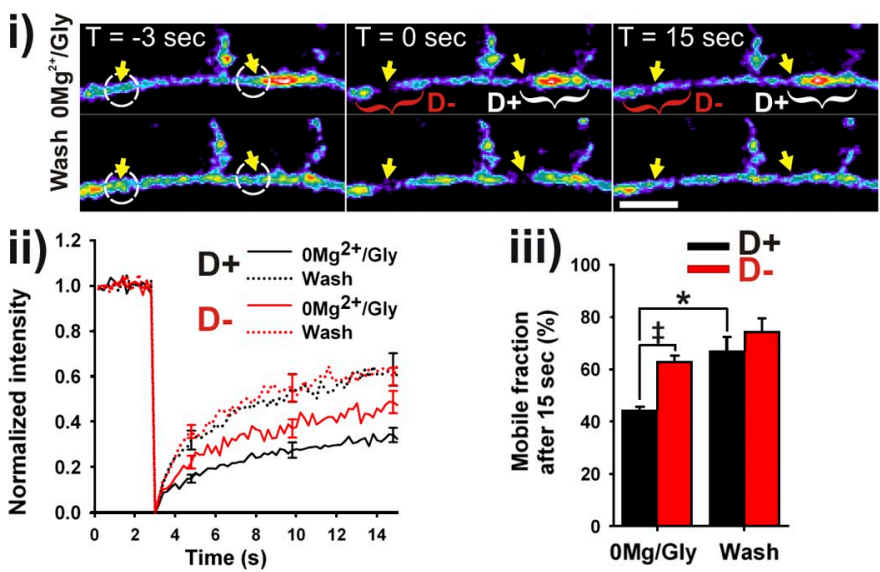
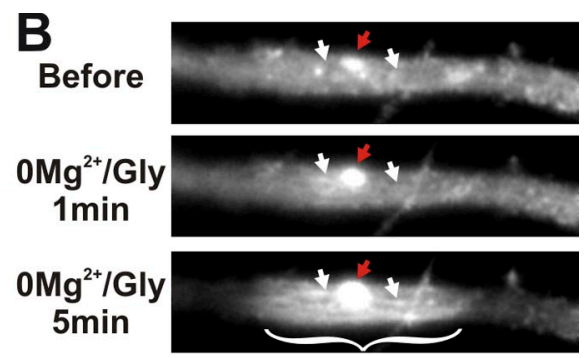

Wash

$30 \mathrm{~min}$
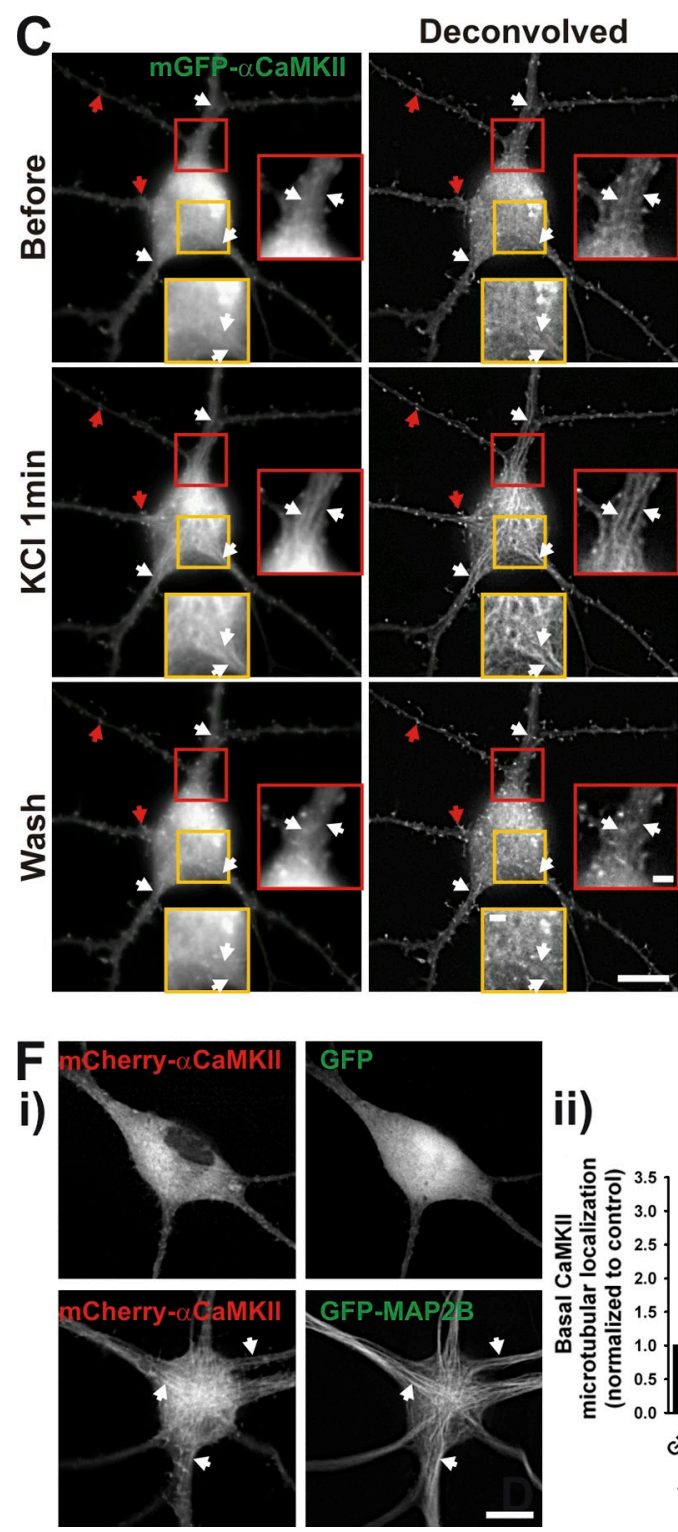

ii)

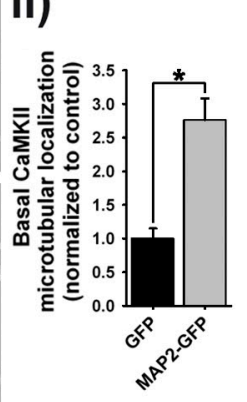

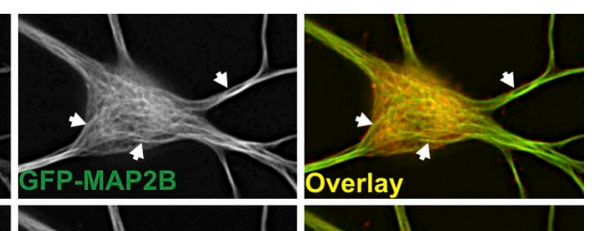
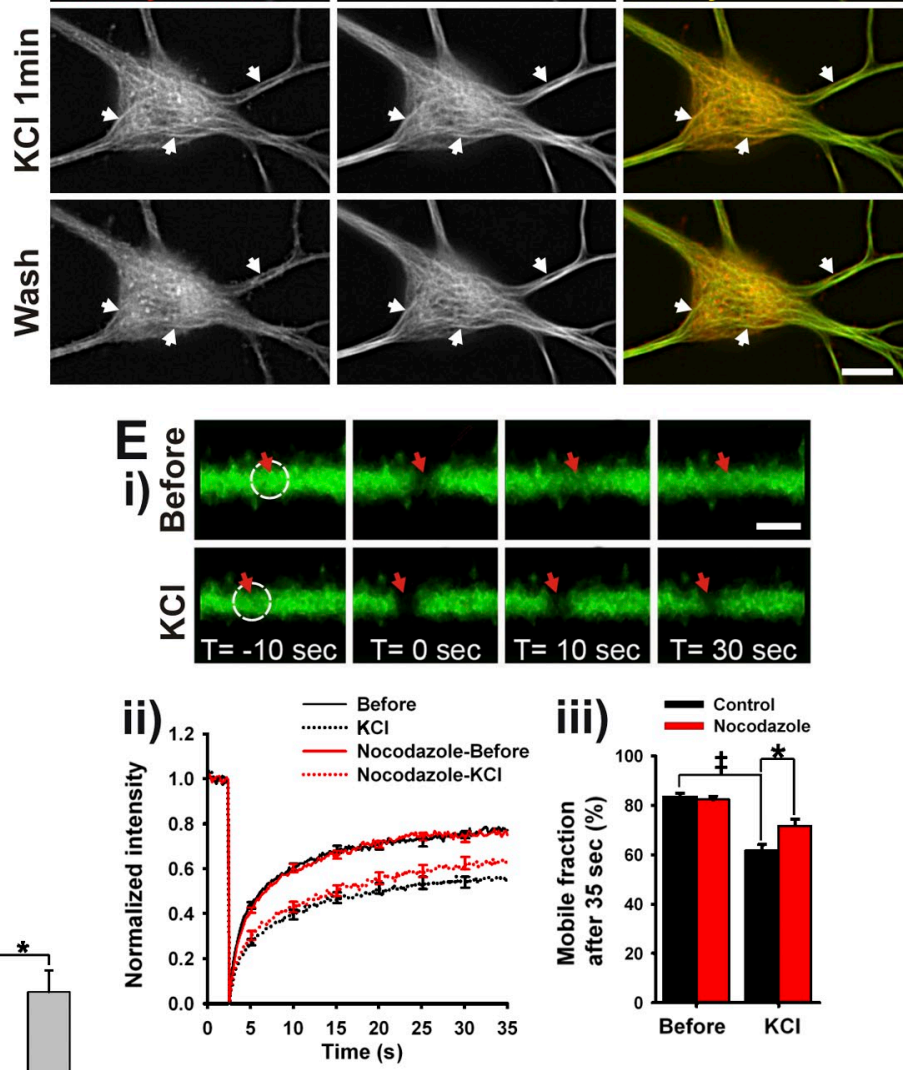

Figure 3. Activity-dependent translocation of $\alpha$ CaMKII to microtubules. (Ai) Time series of FRAP of $m$ GFP- $\alpha$ CaMKII during a OMg ${ }^{2+} / \mathrm{Gly}$ stimulation in a dendrite, either where CaMKII has concentrated ( $D+$, white brackets) or not ( $D-$, red brackets). White circles indicate regions of photobleaching. Bar, $5 \mathrm{~mm}$. (Aii) FRAP curves (bleaching at $\mathrm{t}=3 \mathrm{~s}$ ) and (Aiii) mobile fractions of CaMKIl during and after a $\mathrm{OMg}^{2+} / \mathrm{Gly}$ stimulation in corresponding regions. (B) Dendrite from a neuron expressing mGFP- $\alpha$ CaMKII stimulated with $0 \mathrm{Mg}^{2+} / \mathrm{Gly}$ for $5 \mathrm{~min}$. Bar, $5 \mu \mathrm{m}$. (C-Di) Time-lapse imaging of $\mathrm{mGFP- \alpha CaMKIl}$ (C) or $m$ Cherry- $\alpha$ CaMKII and GFP-MAP2B (D) during a 1-min stimulation with KCl. Bars: (neurons) $10 \mu m$; (inset) $2 \mu m$. (Ei) Time series of FRAP of mGFP- $\alpha$ CaMKII before (top) and during (bottom) a 1-min KCl stimulation. White circles indicate regions of photobleaching. Bar, 3 pm. (Eii) FRAP curves and (Eiii) mobile fractions before and during the stimulation on neurons treated or not with $5 \mu \mathrm{M}$ nocodazole. Arrows in $\mathrm{B}, \mathrm{C}$, and $\mathrm{D}$ point to synaptic (red) or microtubule-like 
that blocked synaptic translocation (Bayer et al., 2001, 2006) also blocked the microtubular translocation (Fig. 4 D).

On the other hand, this analysis revealed differences between the mechanisms of microtubular and postsynaptic translocation of CaMKII. The mutation E96K near the S-site kinase domain did not translocate to microtubules, but still translocated to synaptic sites. Surprisingly, two catalytically dead mutants of CaMKII, K42M and K42R, behaved differently. K42M was clearly already bound to microtubules at basal state, but not to postsynaptic sites, and stimulating the neurons did not lead to more microtubular localization (Fig. 4, C and D). Inversely, K42R did not bind microtubular domains, even after stimulation (Fig. 4, C and D), but did translocate to postsynaptic sites (Shen and Meyer, 1999). These results indicate that although the activity-dependent binding of CaMKII to microtubules bares significant similarities to that of postsynaptic sites, they nonetheless are different (Shen and Meyer, 1999; Marsden et al., 2010). The binding partners for CaMKII on microtubules versus postsynaptic sites are likely different, which may also explain the differences observed in the dissociation of the enzyme from both sites after stimulation (Fig. 1 Biv).

\section{Activity of CaMKII in subdendritic}

hot spots

We next asked whether the CaMKII enriched in dendritic domains is engaged in signaling by measuring its autophosphorylated state at T286. Immunolabeling for phospho-T286 CaMKII revealed that the enzyme was indeed significantly more phosphorylated in dendritic segments where it was enriched compared with segments where it was not (Fig. 5, A and B). In larger dendrites of depolarized neurons, we observed autophosphorylated CaMKII decorating microtubular domains (Fig. 5 C). This observation indicates that CaMKII subdendritic accumulation coincides with increased local signaling of the enzyme.

\section{Subdendritic translocation of $\alpha$ CaMKII correlates with a marked increase in synaptic GluA1-containing AMPARs}

Because of the implication of CaMKII in dendritic exocytosis, cargo release and driving AMPARs to synapses (Maletic-Savatic et al., 1998; Hayashi et al., 2000; Karcher et al., 2001; Correia et al., 2008; Guillaud et al., 2008), as well as its renowned role in synaptic plasticity (Lisman et al., 2012), the dendritic translocation of the enzyme might allow for input-specific targeting of material that would be essential for such plasticity. Using the same culture preparation and cLTP protocol, we showed previously that CaMKII is involved in the activity-dependent synaptic recruitment of GluA1 tagged with $\mathrm{pH}$-sensitive GFP (SEP-GluA1; Opazo et al., 2010). If the dendritic recruitment of CaMKII near active spines revealed here has an impact on this process, we would expect that it correlates with the recruitment of synaptic AMPARs. We therefore imaged SEP-GluA1 at synapses together with mCherry- $\alpha$ CaMKII to identify regions of synaptic and dendritic CaMKII translocation. We observed no significant AMPAR insertion at synapses that did not show synaptic CaMKII accumulation $(\mathrm{S}-$ ) during the stimulation (Fig. 6). By contrast, synapses exhibiting CaMKII accumulation $(\mathrm{S}+$ ) had increased levels of SEP-GluA1. However, within this category of CaMKII-positive synapses, we observed significantly more SEP-GluA1 in those associated with locally increased dendritic CaMKII (D+) compared with those without such increase (D-; Fig. 6). These results support a role for dendritic translocation of CaMKII in promoting further recruitment of AMPARs at synapses.

\section{Subdendritic translocation of $\alpha$ CaMKII correlates with spine plasticity}

In addition to its role in activity- and NMDAR-dependent accumulation of AMPARs to postsynaptic sites during LTP, CaMKII has been shown to promote spine volume increase (Matsuzaki et al., 2004; Xie et al., 2007; Yamagata et al., 2009; Pi et al., 2010) and the formation of new spines and filopodia (Toni et al., 1999; Jourdain et al., 2003). We thus also examined the possible correlation between dendritic CaMKII translocation and spine remodeling by cotransfecting neurons with mCherry (as a structural marker) and mGFP- $\alpha$ CaMKII. Fig. 7 A shows an example of a spine growth in which CaMKII translocates to the spine before accumulating at the spine base during a $0 \mathrm{Mg}^{2+} / \mathrm{Gly}$ stimulation (Video 7). We quantified the changes in the number of spines and filopodia per dendritic segment $(15 \mu \mathrm{m})$, containing (D+) or not (D-) dendritic hot spots of CaMKII, 30 min after stimulation. Although synaptic translocation occurred in both $\mathrm{D}+$ and D - segments, we observed an increase in the number of protrusions in dendritic segments associated with increased CaMKII (Fig. 7 B), leading, 30 min later, to a greater increase in the number of dendritic protrusions compared with neighboring dendritic segments devoid of CaMKII accumulation (1.53 [D+] vs. 1.23 [D-]; Fig. 7 C). Interestingly, this differential increase in spine number in regions associated with dendritic CaMKII accumulation was partly due to a significant increase in the number of mushroom spines, which were $35 \%$ of all spines (Fig. 7 D). Thus, both spine formation and remodeling correlate with the local accumulation of CaMKII in adjacent dendritic regions. These results are consistent with the notion that CaMKII signaling on dendritic microtubules might regulate the local development and plasticity of synapses.

\section{$\alpha$ CaMKII-E96K impairs the activity- \\ dependent increase in synaptic GluA1 and spine remodeling}

To demonstrate causality between CaMKII dendritic translocation and synaptic plasticity, we needed to replace CaMKII in the

sites (white) of CaMKII translocation. ${ }^{*}, \mathrm{P}<0.05$ unpaired $t$ test; $\ddagger, \mathrm{P}<0.05$ paired $t$ test. (Fi) Neuron expressing mCherry- $\alpha$ CaMKII and GFP (top) or GFP-MAP2B (bottom). White arrows point to detectable fibers in CaMKII signal. Bar, $10 \mu \mathrm{m}$. (Fii) Quantification of $\mathrm{mCherry- \alpha CaMKII} \mathrm{microtubular}$ localization in high $\mathrm{Mg}^{2+} /$ low $\mathrm{Ca}^{2+}$ solution when cotransfected with GFP or GFP-MAP2B. $n=49-51$ neurons per condition. ${ }^{*}, \mathrm{P}<0.05$ unpaired $t$ test. See also Figs. S2, S3, S4 and S5 A, and Video 5 and 6. 
Figure 4. Mutagenesis analysis of CaMKI translocation to microtubules. (A) Mean $( \pm S E M)$ $\Delta F / F_{0}$ of $G C a M P 2$ and $(B)$ mean $( \pm S E M)$ fold increase in $\mathrm{mGFP}-\alpha \mathrm{CaMKII}$ microtubular translocation (normalized to control without shRNA) with or without coexpression of shRNA against $\alpha$ - and $\beta$ CaMKII isoforms during a 1-min KC stimulation. $n=17-18$ neurons (A) and 5 neurons $(\mathrm{B})$ per condition. ${ }^{*}, \mathrm{P}<0.05$ unpaired $t$ test. (C) Neurons transfected with shRNA against $\alpha$ - and $\beta C a M K I I$ and expressing different shRNA-resistant GFP-CaMKII mutants, before and during a 1-min stimulation with $\mathrm{KCl}$. White arrows point to detectable fibers in CaMKII signal. Bar, $10 \mu \mathrm{m}$. (D) Fold increase in microtubular translocation during the $\mathrm{KCl}$ stimulation for various mutants. $(n=6-24$ neurons per condition, 4 experiments; asterisk indicates different from GFP). (E) Fold increase in the microtubular translocation during a $1-\mathrm{min}$ $\mathrm{KCl}$ stimulation with or without $\mathrm{KN}$ drugs. (Vehicle: $0.1 \%$ DMSO; $n=13-18$ neurons per condition, 3 experiments; asterisk indicates different from vehicle). ${ }^{*}, \mathrm{P}<0.05$ Kruskal-Wallis followed by Tukey's least-significant difference test. See also Fig. S4.
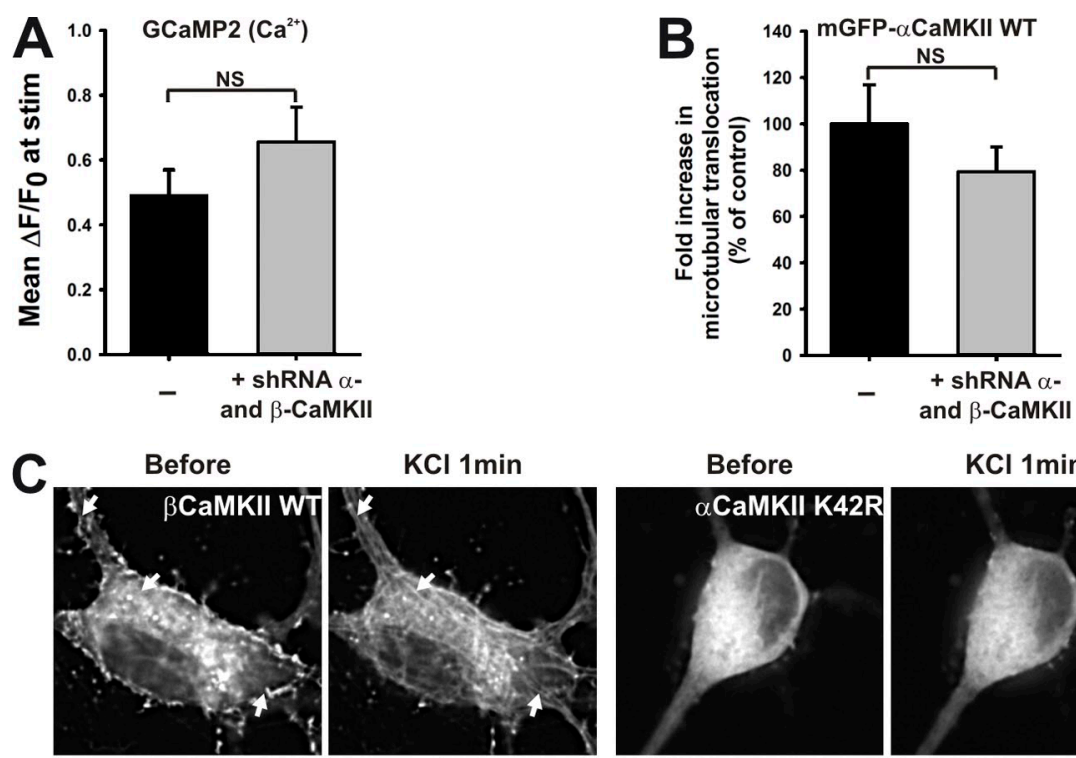

$\mathrm{KCl} 1 \mathrm{~min}$
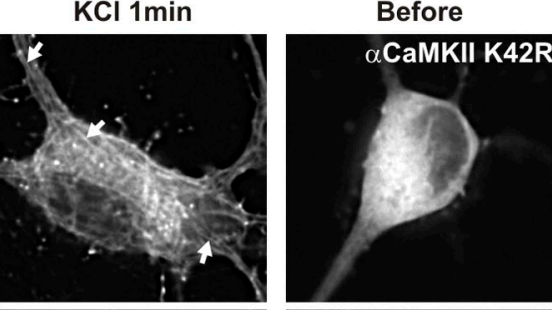

$\mathrm{KCl} 1 \mathrm{~min}$
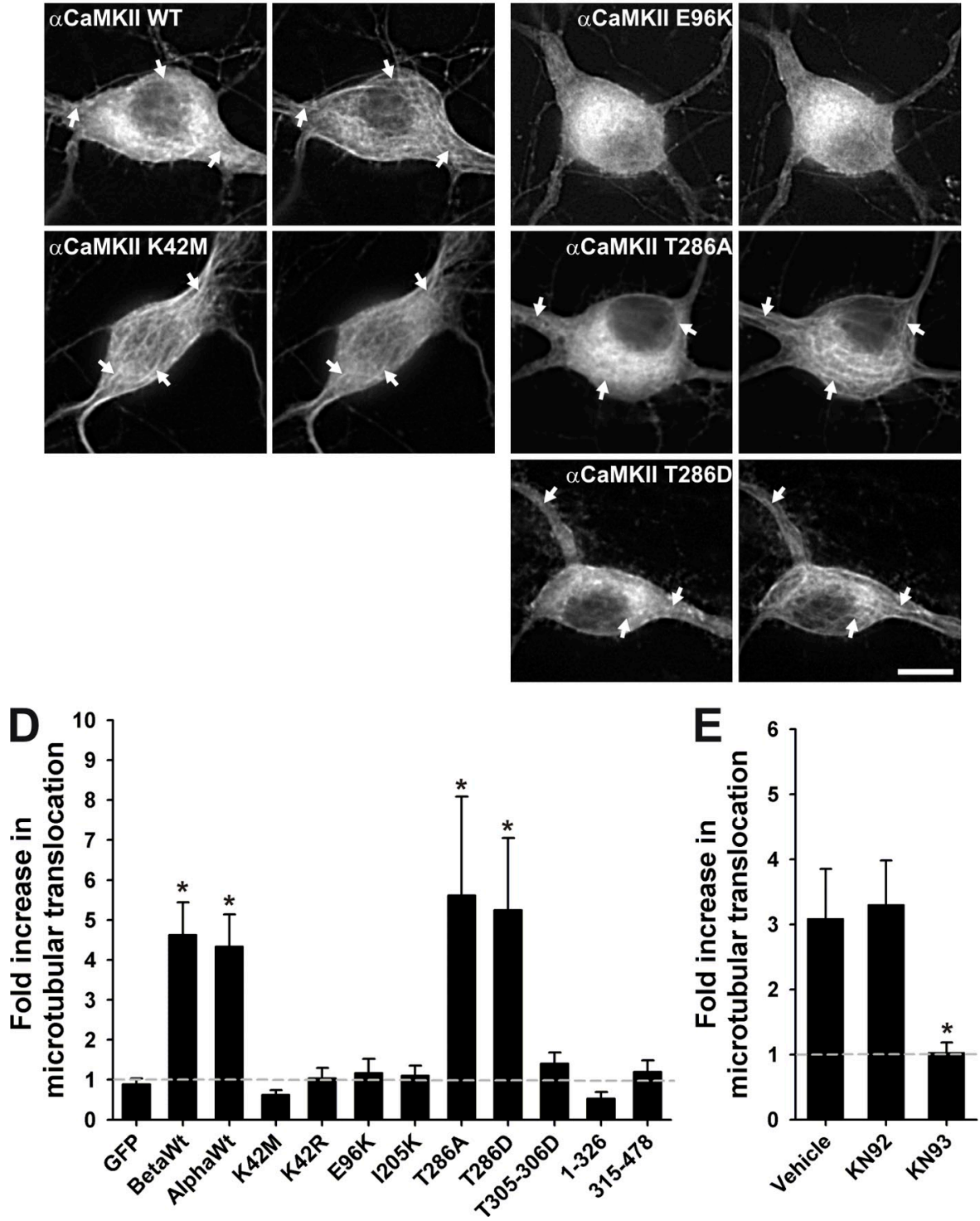
A

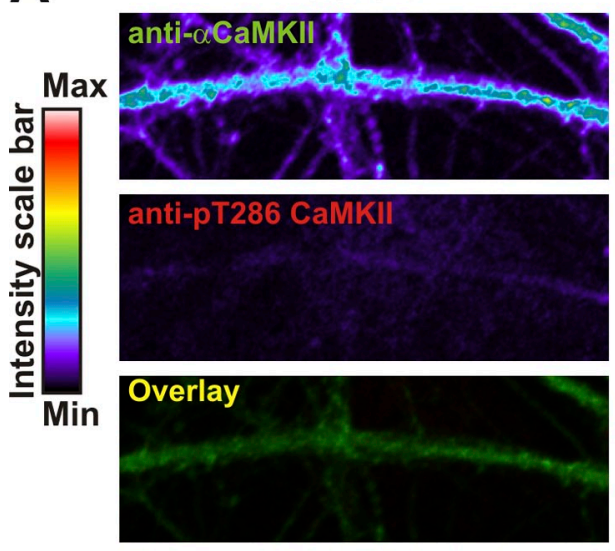

$\mathrm{OMg}^{2+} / \mathrm{Gly}$
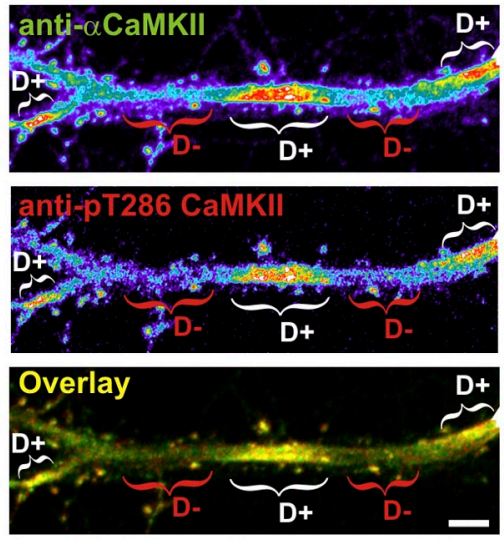

Figure 5. Subdendritic translocation of $\alpha$ CaMKII is associated with a localized increase in CaMKII activity. (A) Dendrite immunolabeled for endogenous $\alpha \mathrm{CaMKIl}$ and phospho-T286 CaMKII in high $\mathrm{Mg}^{2+}$ solution or after a 5-min stimulation with $\mathrm{OMg}^{2+} / \mathrm{Gly}$. Brackets indicate CaMKII dendritic hot spots $(\mathrm{D}+$, white) or adjacent dendritic region ( $D-$, red). (B) Analysis of pT286/ $\alpha$ CaMKII ratio in dendritic regions of neurons as in A. $n=40$ neurons per condition. *, $\mathrm{P}<0.05$ Kruskal-Wallis followed by Tukey's least-significant difference test. (C) Depolarized dendrite exhibits phospho-T286 CaMKII on fiber-like structures. Bars, $5 \mu \mathrm{m}$.

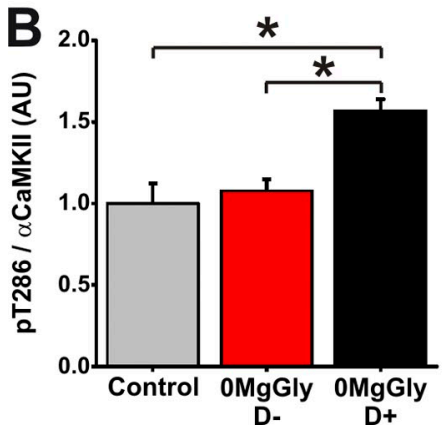

C
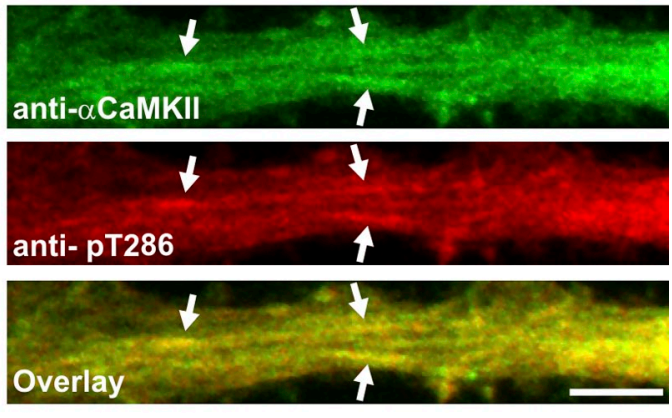

neurons with a mutant that cannot exhibit this translocation, but which retains its catalytic activity and postsynaptic translocation ability. The mutant E96K did not translocate to microtubular domains (Fig. 4, C and D) and did not exhibit subdendritic translocation during a $\mathrm{MMg}^{2+} / \mathrm{Gly}$ stimulation (only 1 hot spot observed out of 8 neurons compared with a mean of 7 events per neuron for WT CaMKII), but could translocate to synapses as much as the WT (Fig. $8 \mathrm{~A}$ ). This mutation is known to maintain kinase activity (Yang and Schulman, 1999), which we confirmed in HEK293 cells using the activity reporter CFP-cVim (Fig. 8 B). We thus tested the impact of replacing endogenous $\alpha$ CaMKII by shRNA with this E96K mutant (resistant to the shRNA) on the synaptic recruitment of SEP-GluA1 and spine growth. Fig. 8, C and D, show that after synaptic stimulation, neurons expressing mCherry- $\alpha$ CaMKII-E96K exhibited significantly less insertion of surface SEP-GluA1 at their synapses and extended less spines as compared with neurons expressing mCherry- $\alpha$ CaMKII WT. These results reinforce the proposal that localized dendritic CaMKII translocation plays a role in synaptic remodeling of nearby synapses.

\section{iscussion}

In Fig. 9, we present a model describing a possible mechanism by which CaMKII might transduce synaptic activity down to the adjacent dendrite, where local processes would take place to support input-specific spine plasticity and insertion of AMPARs. The recruitment of CaMKII to segments of dendrites may also serve to translate the dendritic $\mathrm{Ca}^{2+}$ spikes evoked by clustered inputs (Chalifoux and Carter, 2011;
Kleindienst et al., 2011; Takahashi et al., 2012). We also observed local increases in dendritic $\mathrm{Ca}^{2+}$ during synaptic stimulation in our culture preparation. Dendritic CaMKII accumulation coincided spatially and temporally with these local $\mathrm{Ca}^{2+}$ elevations. The NMDAR-dependent increase in CaMKII to dendritic segments was partly due to intracellular store release of $\mathrm{Ca}^{2+}$ because blocking them with CPA reduced CaMKII subdendritic accumulation. This is consistent with the observation that CPA reduced synaptically evoked, NMDAR-dependent $\mathrm{Ca}^{2+}$ transients (Emptage et al., 1999). Blocking $\mathrm{Na}^{2+}$ and $\mathrm{Ca}^{2+}$ channels reduced dendritic CaMKII accumulation, probably by blocking evoked release of glutamate as well as back-propagating action potentials. The necessity for a confined $\mathrm{Ca}^{2+}$ gradient to trigger the localized dendritic translocation of the kinase likely explains why we and other groups (Shen and Meyer, 1999; Otmakhov et al., 2004; Hudmon et al., 2005; Bayer et al., 2006) have not observed this process when imaging mGFP-CaMKII with bath application of glutamate or NMDA, which causes a global increase in intracellular $\mathrm{Ca}^{2+}$ and the translocation of the kinase to microtubular domains throughout the dendrites. Glutamate uncaging onto single spines has been shown to cause only low $\mathrm{Ca}^{2+}$ elevation in their dendritic base (Sobczyk et al., 2005; Higley and Sabatini, 2008) and has not been associated with CaMKII accumulation at the spine base (Lee et al., 2009). However, dendritic depolarization by retrograde action potentials coupled with synaptic stimulation should synergistically induce a higher level of dendritic $\mathrm{Ca}^{2+}$ (Higley and Sabatini, 2008) that may involve NMDA spikes (Schiller et al., 1998, 2000; Chalifoux and Carter, 2011), which would favor localized dendritic translocation of CaMKII. 
A
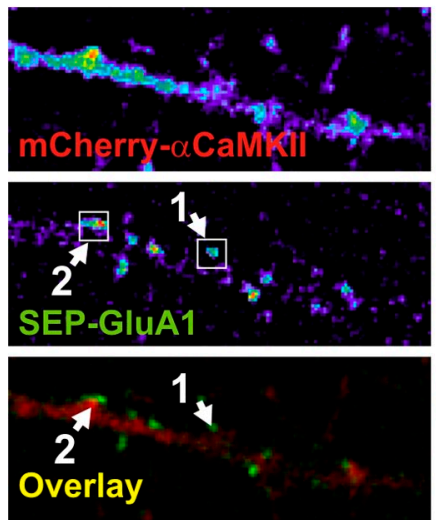

B

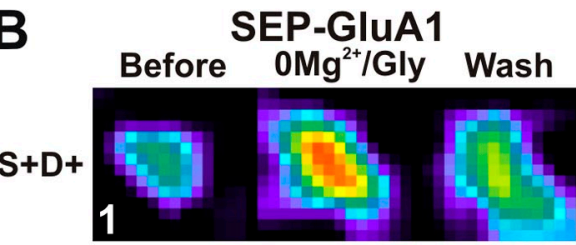

$\mathrm{S}+\mathrm{D}$

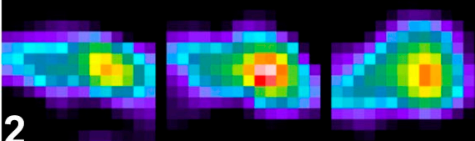

S.
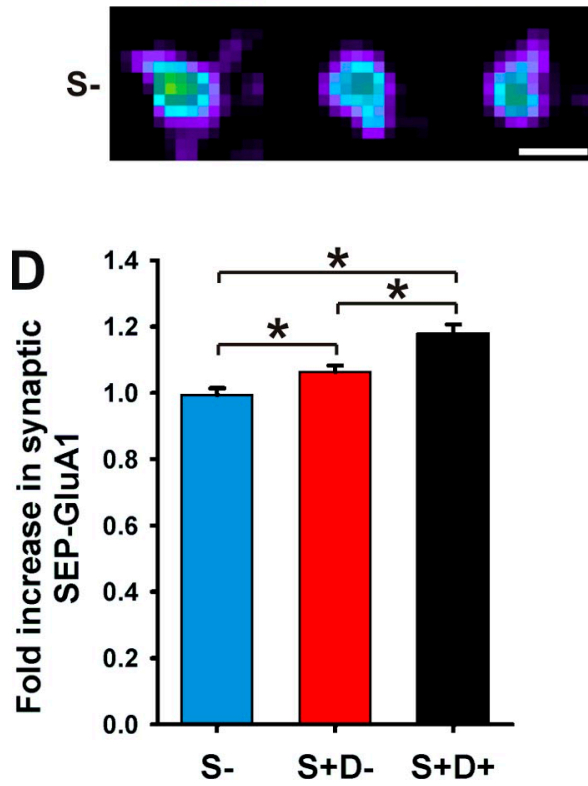

$\mathrm{OMg}^{2+} /$ Gly $5 \mathrm{~min}$
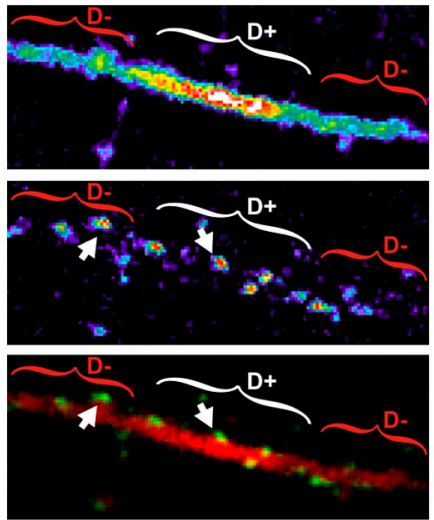

Wash 10min
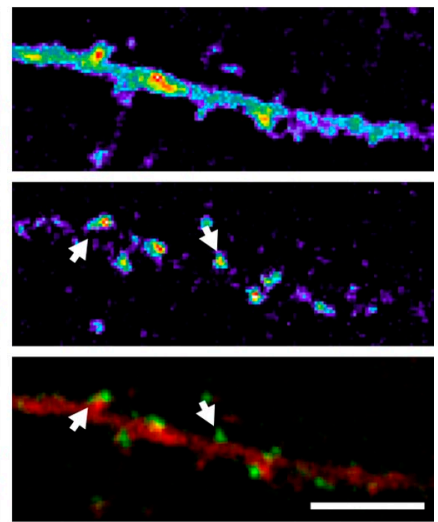
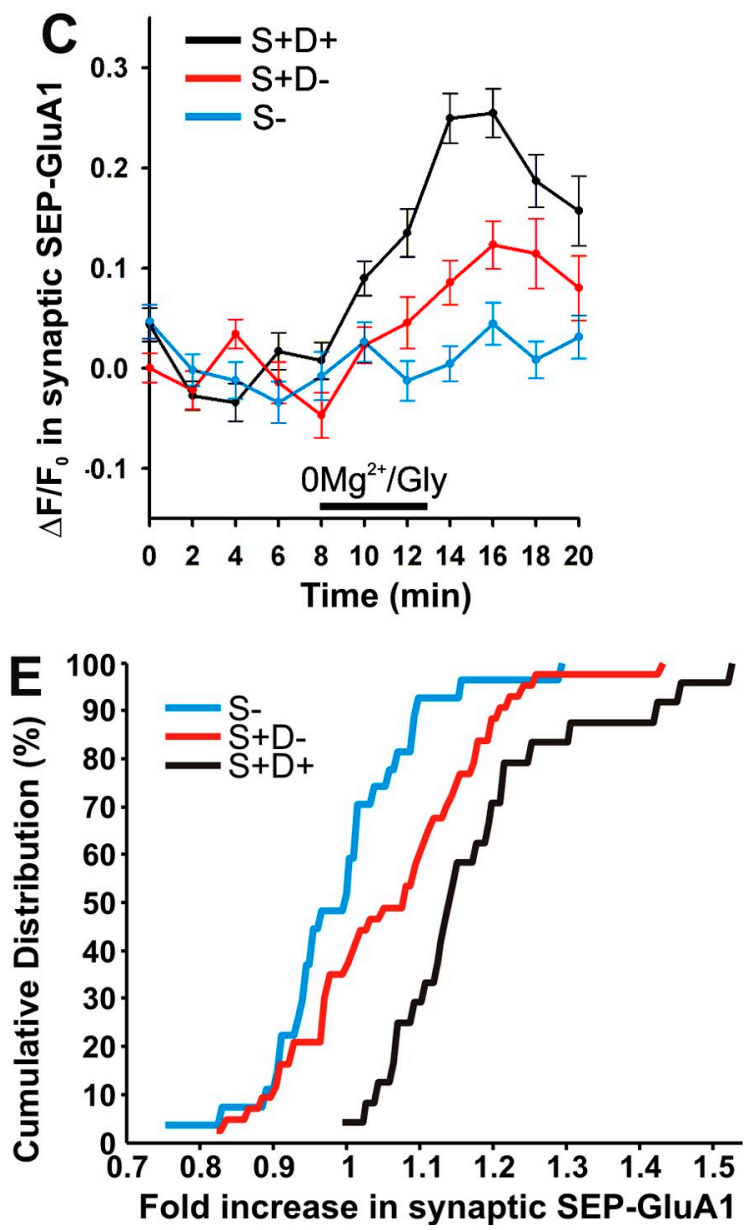

Figure 6. Subdendritic translocation of $\alpha \mathrm{CaMKIl}$ correlates with an increase of surface GluAl at neighboring postsynaptic sites. (A) Dendrite coexpressing $\mathrm{mCherry- \alpha CaMKII}$ and SEP-GluAl stimulated with $0 \mathrm{Mg}^{2+} / \mathrm{Gly}$ for $5 \mathrm{~min}$. Brackets indicate CaMKIl dendritic hot spots (D+, white) or adjacent dendritic region ( $D-$, red). Arrows point to a spine near (1) or adjacent (2) to an mCherry- $\alpha$ CaMKII dendritic hot spot. ( $n=7$ neurons, $24-43$ synapses per group). Bar, 5 m. (B) Representative examples of SEP-GluA1 intensity at postsynaptic sites for synapses without (blue, $S-$ ) or with (S+) synaptic CaMKIl translocation, which are near (black, S+D+) or not (red, $\mathrm{S}+\mathrm{D}-$ ) a CaMKIl dendritic hot spot. Synapses 1 and 2 are pointed in A. Bar, 0.5 um. (C-E) Time-lapse analysis (C), histogram (D), and cumulative distribution (E) of the increase in SEP-GluAl for synapses in the same category as described in B. ${ }^{*}, P<0.05$ Anova followed by Tukey's least-significant difference test.

Accumulation of endogenous CaMKII in dendritic sections, independently of protein synthesis, was in fact observed in hippocampal slices by activating specific lamina with trains of high-frequency stimuli during a few minutes (Steward and Halpain, 1999). Using trains of local application of Glu/Gly, we observed a localized dendritic accumulation of CaMKII, which coincided spatially and temporally with the confined $\mathrm{Ca}^{2+}$ increase. This is in contrast with what Rose et al. (2009) observed with a single 10-25-ms puff evoking the delayed synaptic translocation of the enzyme throughout the entire somatodendritic area of neurons. We never observed this phenomenon upon local puffs of Glu/Gly. The reasons for this difference are unclear. 

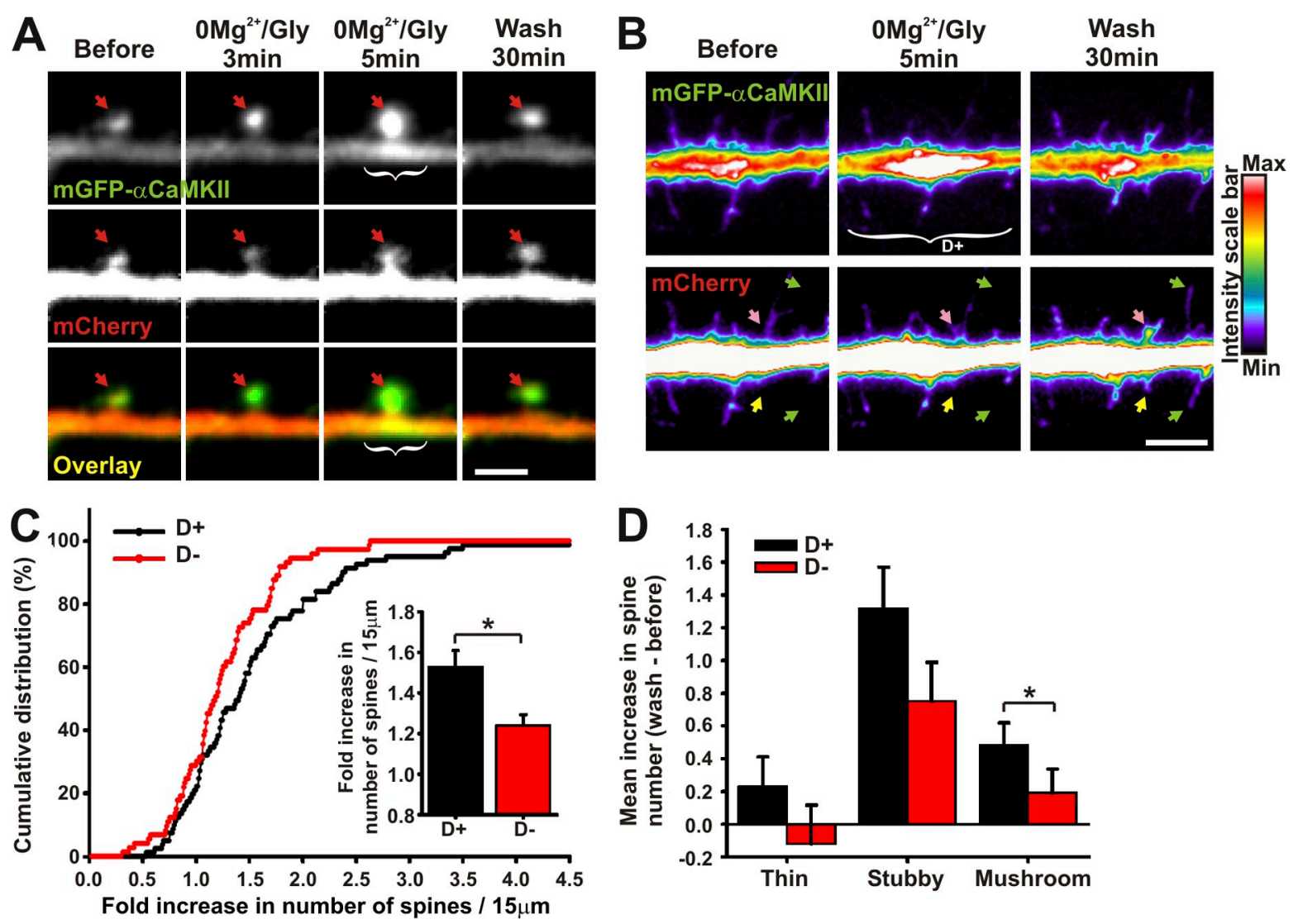

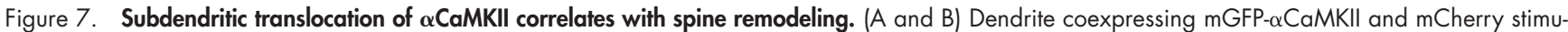
lated with $\mathrm{OMg}^{2+} / \mathrm{Gly}$ for $5 \mathrm{~min}$. Arrows point to spines (red), spine morphological change (pink), new protrusion (yellow), and spine elongation (green). White brackets indicate regions of dendritic CaMKII hot spot (D+). (C) Cumulative distribution and associated histogram of the fold increase in the number of spines on 15- $\mathrm{m}$ dendritic sections, associated with regions revealing dendritic CaMKIl accumulation (D+) or not (D-). (D) Histogram of the mean \pm SEM increase in spine number classified by spine morphology and associated with regions revealing dendritic CaMKII accumulation $(D+)$ or not $(D-)$. $(n=19$

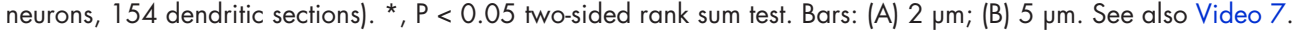

In contrast to our observations, Rose et al. (2009) reported a global $\mathrm{Ca}^{2+}$ elevation in response to their local stimulation, which may have set in motion a different type of cell-wide response (Branco and Häusser, 2010).

Our results suggest that $\mathrm{Ca}^{2+}$ elevation causes CaMKII binding to microtubules and/or associated proteins. Any cellular organelle, cargo, or neurofilaments linked to microtubules could be targets for CaMKII binding. The list of known CaMKII substrates associated with microtubules, such as MAP2, Tau, MAP6, kinesins, and neurofilaments, to name a few (Schulman, 1984; Baudier and Cole, 1987; Hanson and Schulman, 1992; Baratier et al., 2006), is growing. It is likely that many of these support the rapid and reversible recruitment of the enzyme to microtubular domains upon $\mathrm{Ca}^{2+}$ elevation. In fact, overexpression of MAP2 increased the basal decoration of microtubular domains by CaMKII. Although this result might support MAP2 as a strong candidate, the overexpression of MAP2 could have also further stabilized microtubules and hence favored additional CaMKII-binding interactions.

On the kinase side, the mechanism of binding to microtubules bears similarities and differences in comparison to that of postsynaptic sites. Kinase activation, i.e., the displacement of the inhibitory domain but not its activity, per se, is required for both sites, as it is blocked by KN93 and by mutating T305/306 to D. Thus, displacement of the auto-inhibitory domain is likely to expose the required binding sites on the kinase.

The case of $\mathrm{K} 42 \mathrm{M}$ is intriguing as it behaves differently than K42R, two mutants initially generated to prevent CaMKII catalytic activity by interfering with ATP binding (Hanson et al., 1994). It is possible that replacing the lysine with a methionine changes the enzyme conformation more drastically than the arginine, which retains the positive charge.

The unbinding kinetics observed between dendritic and postsynaptic translocation are distinct. We and Bayer et al. (2001, 2006) proposed earlier that the persistent binding of CaMKII to postsynaptic sites, after its activity-dependent translocation, is mediated through a tight interaction between the T-site of the kinase and the cytoplasmic tail of the NMDA receptor subunit GluN2B. By contrast, the microtubular binding did not show persistence after stimulation. The T-site nevertheless appears to be involved because the $\mathrm{I} 205 \mathrm{~K}$ mutant did not show microtubular translocation.

The multimeric arrangement of CaMKII is required for both sites of translocation, suggesting that binding of the enzyme is favored by multivalent interactions rather than a single binding interaction as in the monomeric mutant (1-326). Autophosphorylation at T286 seems to have little effect on dendritic translocation, whereas it can modulate its translocation 
A

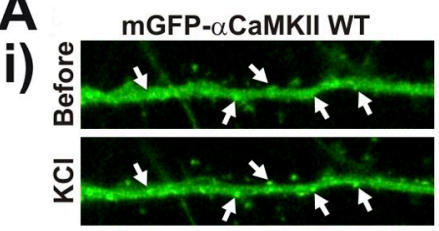

mGFP- $\alpha$ CaMKII E96K

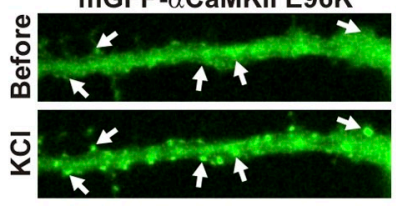

Homer 1c-DsRed

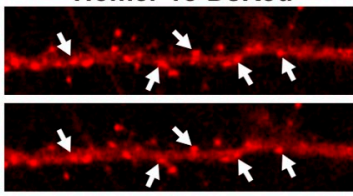

Homer 1c-DsRed

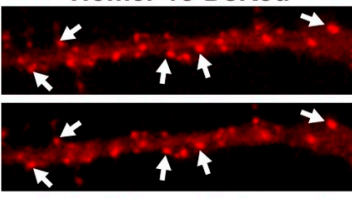

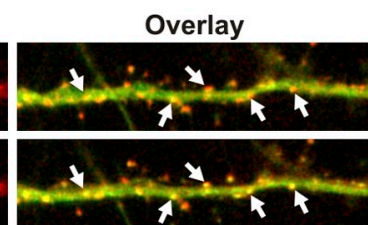

Overlay

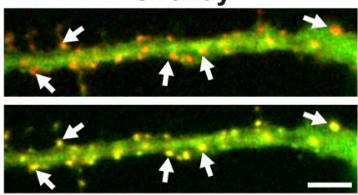

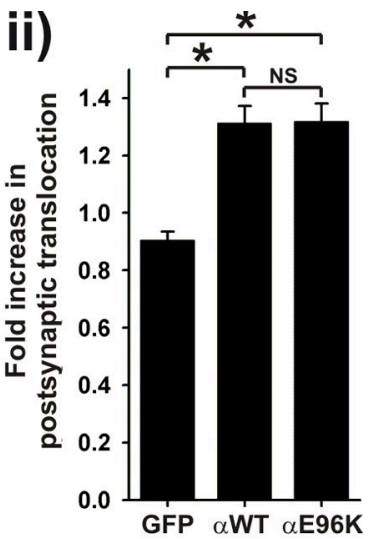

B

lono+

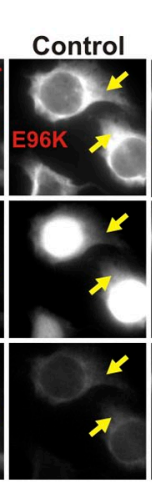

Iono+

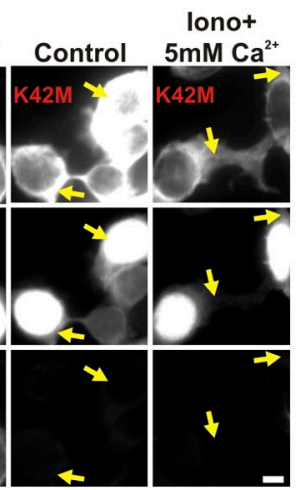

ii) = ${ }_{\text {lono }+5 \mathrm{mM} \mathrm{Ca}}^{\text {Catrol }}$
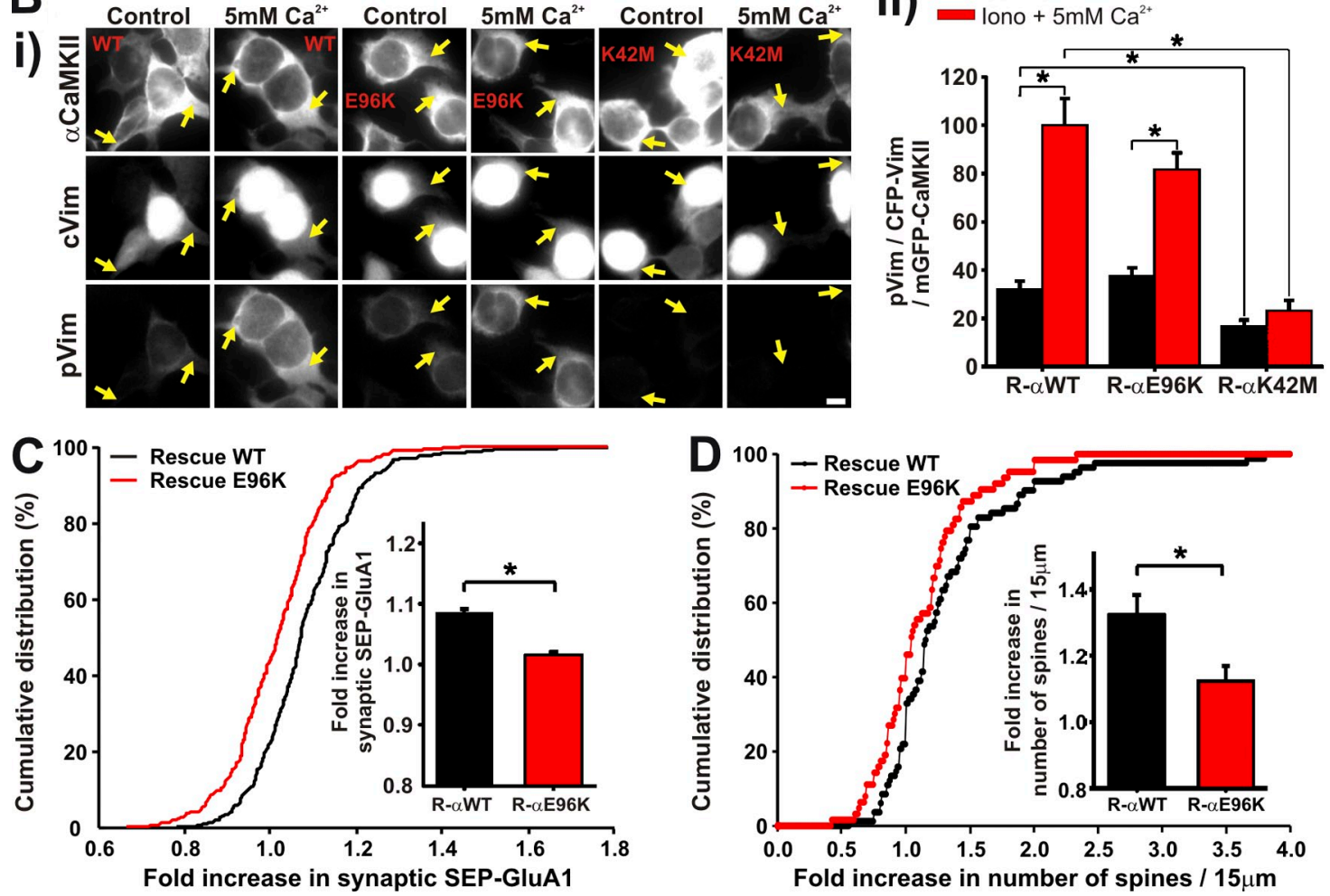

Figure 8. E96K, an active mutant of $\alpha \mathrm{CaMKII}$ that translocates to synapses, but not to microtubules, impairs activity-dependent increase in surface GluAl synaptic insertion and spine remodeling. (Ai) Neurons transfected with mGFP- $\alpha$ CaMKII WT or E96K and Homer l c-DsRed and stimulated with KCl for 1 min. (ii) Fold increase in CaMKII postsynaptic translocation, cotransfected or not with Homer lc-DsRed during the KCl stimulation. Because CaMKII postsynaptic translocation was not different with or without Homer lc coexpression, all neurons were pooled together $(n=13-22$ neurons per condition, 6 experiments). (Bi) HEK cells cotransfected with mGFP- $\alpha$ CaMKII WT, E96K or K42M, and CFP-cVim. The cells were stimulated (HBSS $+10 \mu M$ ionomycin $+5 \mathrm{mM} \mathrm{Ca}{ }^{2+}$ ) or not (HBSS) for $5 \mathrm{~min}$, fixed, and immunolabeled for phospho-Vim. (Bii) Quantification of cVim phosphorylation by $\alpha$ CaMKII WT, E96K, and K42M. The intensity of pVim immunolabeled was divided by the intensity of both total cVim and CaMKII. $n=351-536$ cells per condition, 2 experiments. Bars, $5 \mu m$. $(A$ and $B) *, P<0.05$ Kruskal-Wallis followed by Tukey's least-significant difference test. (C) Histogram and cumulative distribution of the fold increase in SEP-GluAl for synapses of neurons with synaptic translocation $(\mathrm{S}+$ ) of mCherry- $\alpha \mathrm{CaMKII} \mathrm{WT}$ or E96K rescue, in the presence of shRNA against $\alpha$ CaMKII, during a stimulation with $\mathrm{OMg}^{2+} / \mathrm{Gly}$ for $5 \mathrm{~min}$. ( $n=8$ neurons, 272-300 synapses per group). (D) Histogram and cumulative distribution of the fold increase in the number of spines on 15- $\mu \mathrm{m}$ dendritic sections in neurons expressing mGFP- $\alpha$ CaMKII WT or E96K rescue as well as mCherry and shRNA against $\alpha$ CaMKII. ( $n=6-8$ neurons, $63-82$ dendritic sections). (C and $D){ }^{*}, P<0.05$ two-sided rank sum test.

to excitatory synapses (Bayer et al., 2001, 2006; Hudmon et al., 2005) and is required for translocation to inhibitory synapses (Marsden et al., 2010). To date, of all the active mutants tested, only the mutation E96K did not exhibit dendritic translocation, while it did to synapses. This mutation is on the edge of the catalytic site of the enzyme, but does not prevent substrate phosphorylation as measured in vitro (Yang and Schulman, 1999) and in cells. It may, however, affect the transient binding to some substrates. In fact, transient binding to microtubular substrates may be what supports the dendritic relocation that we have observed. Further investigation is needed to dissect the detailed mechanism of CaMKII microtubular translocation.

What are, then, the functions of CaMKII binding to microtubular domains? They likely depend on the localization of this binding, such as the soma, dendrites, the axon, or even the growth cone, and on the activity pattern that leads to this interaction. 


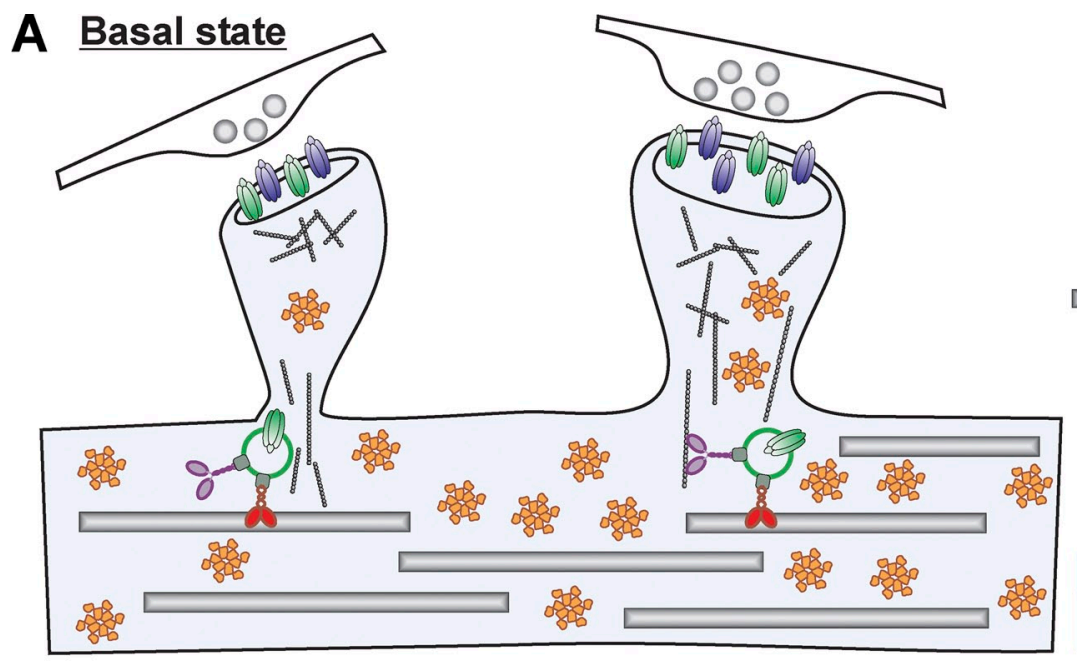

\section{- Glutamatergic presynaptic vesicle \\ AMPA receptor \\ NMDA receptor \\ Actin filaments \\ Microtubules \\ CaMKII \\ 8- Myosin V \\ Kinesin \\ - Adaptor protein}

Low $\mathrm{Ca}^{2+}$

High $\mathrm{Ca}^{2+}$

\section{B High synaptic activity}

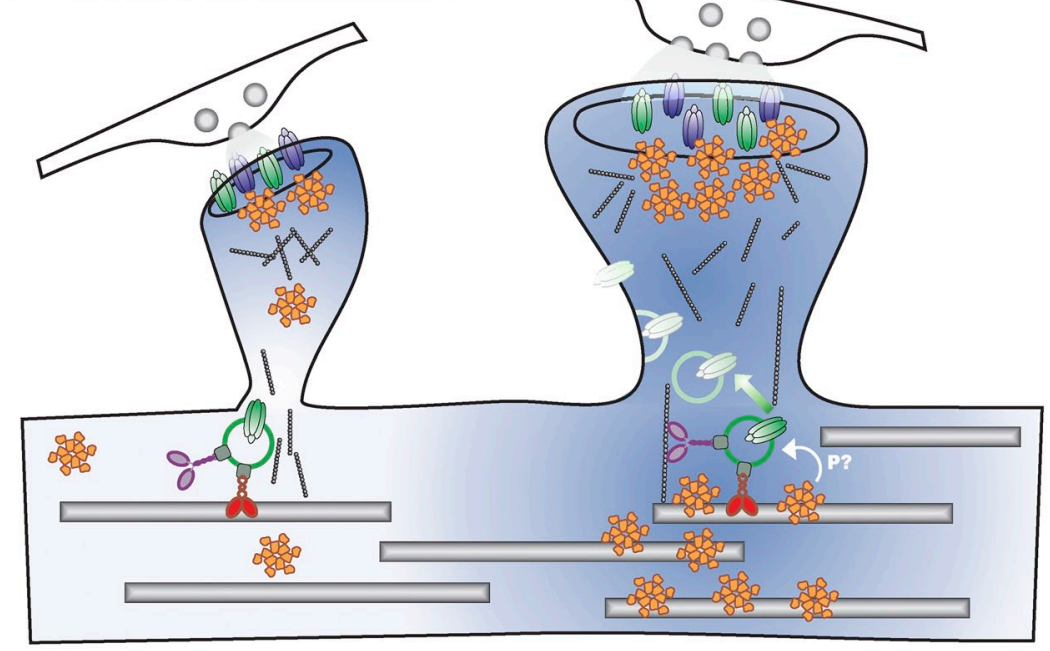

\section{Post-activation}

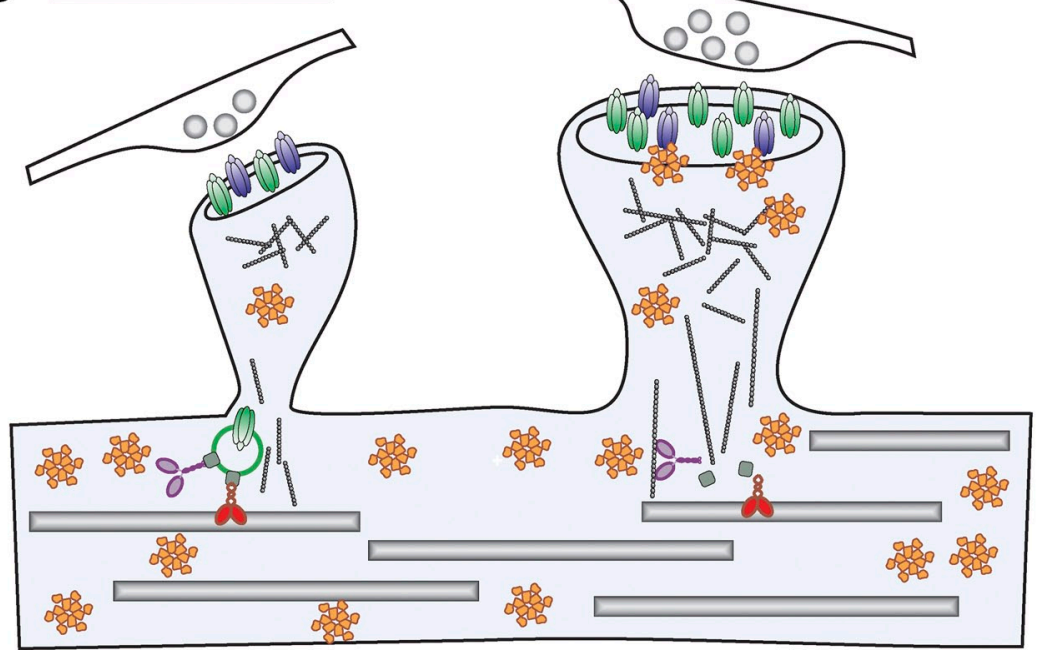

Figure 9. Model of CaMKII subdendritic translocation and its impact on synaptic plasticity. (A) During basal activity, intracellular free $\mathrm{Ca}^{2+}$ is low and a large fraction of CaMKII freely diffuses throughout the neuron. (B) During elevated synaptic activity, $\mathrm{Ca}^{2+}$ increases in spines and in nearby dendritic compartments. CaMKII translocates to activated synapses and subdendritic regions by binding to synaptic and microtubular partners, respectively. This local accumulation where $\mathrm{Ca}^{2+}$ activity is high leads to a depletion of the enzyme concentration on adjacent segments where $\mathrm{Ca}^{2+}$ activity is lower. The localized dendritic activation of CaMKII leads to a confined phosphorylation of substrates in this region. Hypothetically, CaMKII target may be proteins involved in receptor trafficking. Cargos containing AMPARs could then be released by CaMKII phosphorylation, thereby facilitating their delivery to activated synapses. (C) After activation, CaMKII largely unbinds from microtubules but only partially from strongly activated synapses, which remain larger and retain more AMPARs. 
In the case of global, high frequency firing of the neuron, leading to global free $\mathrm{Ca}^{2+}$ elevation, we would expect the enzyme to bind throughout the microtubular network, and thereby transmit information about the activity state of the cell down to its core cytoskeleton. The large number of substrates for CaMKII on microtubules offers multiple downstream functions such as dendritic remodeling, active transport and delivery, and exocytosis. In the case of local synaptic activity into a specific dendritic branch or segment, the binding of CaMKII to microtubular elements is restricted to that subcellular region, leading to local signaling, which might impact on local synaptic remodeling.

We examined two specific features of synaptic remodeling: the recruitment of AMPARs and spine growth. Our results show an enhanced accumulation of GluA1 at synapses where CaMKII accumulated and particularly more in those located near a hot spot of dendritic CaMKII as well. Beyond this correlation, the replacement of endogenous CaMKII by the E96K mutant argues for causality between the localized dendritic translocation and the local plasticity. This enrichment of receptors might be due to their increased exocytosis in the CaMKII-rich dendritic region. Indeed, CaMKII regulates dendritic exocytosis (Maletic-Savatic et al., 1998), the exocytosis of AMPARs was observed in the portion of the dendrite near stimulated spines (Makino and Malinow, 2009; Patterson et al., 2010), and the insertion of new GluA1 in synapses correlates with a dendritic $\mathrm{Ca}^{2+}$ transient (Korkotian and Segal, 2007). Recently, however, the activity-dependent exocytosis of AMPARs, evoked by glutamate uncaging, was shown to require the Ras-ERK pathway, independently of CaMKII (Patterson et al., 2010). Conceivably, the presence of TTX, used in this uncaging protocol, reduced $\mathrm{Ca}^{2+}$ elevation and CaMKII activity in the dendrite, thereby masking a possible contribution of the enzyme in additional GluA1 exocytosis.

Another key function proposed for CaMKII has been the regulation of transport proteins involved in AMPAR trafficking. The kinase might release GluA1, contained in Rab11 endosomes located notably at the base of spines (Park et al., 2006), possibly by phosphorylating myosin V (Karcher et al., 2001). The activity- and myosin V-dependent translocation of recycling endosomes into spines was shown to support both spine enlargement and AMPAR delivery (Wang et al., 2008). Thus, the enrichment of CaMKII at the base of active spines via binding to microtubules provides a possible mechanism to specifically release AMPARs at the appropriate synapse. Clustering of AMPARs into the synaptic cleft can then be facilitated also by CaMKII via the phosphorylation of stargazin $\mathrm{C}$ terminus (Opazo et al., 2010). This phosphorylation could potentially occur on cargo vesicles or at the plasma membrane.

In addition to CaMKII's role in regulating morphology of existing spines, the enzyme plays a role in the formation and elongation of new protrusions during LTP (Toni et al., 1999; Jourdain et al., 2003). Synaptogenesis is accompanied by confined dendritic $\mathrm{Ca}^{2+}$ (Lohmann and Bonhoeffer, 2008), which might support the developmental process through CaMKII. $\beta C a M K I I$ has been linked to filopodial extension and synapse formation (Fink et al., 2003). Because this subunit also exhibits subdendritic translocation, it may have contributed to the effects observed on GluA1 recruitment or spine remodeling in combination with $\alpha$ CaMKII, which would likely accumulate at the same sites. The enzyme might also participate in the activitydependent change in microtubules that affects molecular transport (Maas et al., 2009). Localized CaMKII could also support local translation of mRNA - including its own $\alpha$ CaMKII mRNA, required for plasticity (Sutton and Schuman, 2006).

Our results describe a potential mechanism supporting input-specific synaptic plasticity (Fig. 9). By causing elevation in $\mathrm{Ca}^{2+}$ at their base, active excitatory synapses could then act on the dendritic delivery of material that supports and/or enhances their own plasticity. This mechanism would complement the synaptic tagging and capture hypothesis, for which CaMKII has also been proposed to constitute a key element (Frey and Morris, 1998; Redondo and Morris, 2011). The role as a synaptic tag may be supported by its ability to reside in the PSD for a prolonged time after its translocation (Bayer et al., 2006), coupled to its locked active state when bound to GluN2B (Bayer et al., 2001, 2006), and its multivalent structure favoring multiple interactions with other synaptic partners and scaffolding with other CaMKII holoenzymes (Hudmon et al., 2005; Robison et al., 2005). It should be noted that LTP of an individual spine can be evoked by causing $\mathrm{Ca}^{2+}$ elevation only inside the spine and not in the dendritic shaft, as shown with uncaging approaches (Lee et al., 2009). Thus, dendritic CaMKII is not necessary for certain forms of LTP, although in some cases it might enhance it. In fact, localized synaptic depolarization and/or dendritic spikes have been reported to induce stronger LTP than that induced by back-propagating action potentials (Hardie and Spruston, 2009). These observations suggest that spatial proximity of inputs could favor stronger LTP. Proximity to potentiated synapses helps weaker inputs to generate LTP, but only shortly after (Harvey and Svoboda, 2007); localized and transient dendritic modifications could support such a process, and CaMKII could thus be involved. The proposal that the dendritic branch or segment is the critical site of integration in long-term storage (Govindarajan et al., 2006, 2011) is appealing and the localized dendritic recruitment of CaMKII could be part of the process. CaMKII is a multifunctional enzyme that regulates many aspects of neuronal function and synaptic plasticity. Further studies are needed to better understand the functional consequences of CaMKII recruitment to microtubular domains near activated synapses, but numerous potential implications can be drawn.

\section{Materials and methods}

\section{Rat hippocampal cultures, plasmids, and transfection}

Dissociated hippocampal neurons were prepared as described previously (Hudmon et al., 2005; Nault and De Koninck, 2010). In brief, hippocampi dissected out of P1-P3 rats were dissociated enzymatically (papain, $12 \mathrm{U} / \mathrm{ml}$; Worthington) and mechanically (trituration through Pasteur pipette). After dissociation, the cells were washed, centrifuged, and plated on poly-D-lysine-coated Aclar $(12 \mathrm{~mm})$ or glass $(18 \mathrm{~mm})$ coverslips at a density of $\sim 350-450$ cells $/ \mathrm{mm}^{2}$. Growth media consisted of Neurobasal and B27 (50:1), supplemented with penicillin/streptomycin $(50 \mathrm{U} / \mathrm{ml} ; 50 \mathrm{\mu g} / \mathrm{ml}$ ) and $0.5 \mathrm{mM} \mathrm{L}$-glutamax (Invitrogen). Fetal bovine serum (2\%; Hyclone) was added at time of plating. After $5 \mathrm{~d}$, half of the media was changed without serum and with Ara-C (5 $\mu$ M; Sigma-Aldrich) to limit proliferation of non-neuronal cells. Twice a week thereon, half of the growth medium was replaced with serum- and Ara-C-free medium. 
Neurons were transfected at 11-15 DIV using Lipofectamine 2000 (Invitrogen) as described previously (Hudmon et al., 2005). When shRNA were used, neurons were transfected at 8 DIV and imaged at 11 DIV. $m G F P-\alpha C a M K I I$ and mGFP- $\beta$ CaMKII plasmids contained a mutation (A207K) in the eGFP (Takara Bio Inc.) to eliminate GFP-dependent aggregation (Zacharias et al., 2002; Hudmon et al., 2005). mCherry and $m$ Cherry- $\alpha$ CaMKII plasmids were generated by PCR amplification of the cDNA encoding mCherry from plenti-CaMKII-hChR2-mCherry-WPRE (a gift from Karl Deisseroth, Stanford University, Stanford, CA) with primers 5'-GCGCTACCGGTCGCCACCATGGTGAGCAAGGGCGAGGAGGATAACA-3' and 5'-CTTGTACAGCTCGTCCATGCCGC-3'. The PCR product was then digested with Agel-BsrGl and inserted into the Agel$\mathrm{BsrGl}$ sites of $\mathrm{mGFP}-\mathrm{Cl}$ or $\mathrm{mGFP}-\alpha \mathrm{CaMKII}$. $\alpha$ - and $\beta$ CaMKII shRNAs were obtained from Yasunori Hayashi (RIKEN Brain Science Institute, Saitama, Japan) and Sang Lee (Medical College of Wisconsin, Milwaukee, WI). mGFP- $\alpha$ CaMKII WT-rescue was described previously (Opazo et al., 2010). $m$ GFP- $\beta$ CaMKII WT-rescue was generated by inserting silent mutations on mGFP-BCaMKII WT plasmid: 5'-GAGTACGCTGCAAAAATAA-3' compared with the $\beta$ CaMKII shRNA target sequence: 5'-GAGTATGCAGCTAAGATCA-3'. mGFP- $\alpha$ CaMKII K42M and K42R-rescue were obtained by digestion of $m$ GFP- $\alpha$ CaMKII WT-rescue with Pflml and insertion into the Pflml sites of mGFP- $\alpha$ CaMKII K42M or K42R (generated by mutagenesis). Rescue of $\alpha$ CaMKII E96K, I205K, and T305-306D were generated by mutagenesis to insert the silent mutations and verified by sequence analysis. $\alpha$ CaMKII T286A and T286D rescue were generated by digestion of $m$ GFP- $\alpha \mathrm{CaMKII}$ WT-rescue with $\mathrm{BamHI}-\mathrm{BbvCl}$ and insertion into the BamHI-BbvCl sites in mGFP- $\alpha$ CaMKII T286A and T286D, respectively (Hudmon et al., 2005). Monomeric $\alpha$ CaMKII (aa 1-326) and the association domain (aa $315-478$ ) were subcloned into the mGFP- $\alpha \mathrm{CaMKII}$ backbone using a PfIMl restriction insert (Hudmon et al., 2005). mCherry$\alpha$ CaMKII WT-rescue and E96K-rescue were obtained by replacing mGFP with mCherry using Agel-BsrGI. CFP-cVim-NES was constructed using FC1.2c-Vim CFP-NES (provided by J. Tsui and H. Schulman, Stanford University, Stanford, CA) digested with BamHl and Mfel and introduced into an enhanced GFP vector (Takara Bio Inc.). SEP-GluA1 plasmid was generated by PCR amplification of the cDNA encoding Superecliptic pHluorin (obtained from G. Miesenbock, University of Oxford, Oxford, UK) with primers 5'-GTCATCGCTAGCATGAGTAAAGGAGAAGAACTT-3' and 5'-GATGCACGTACGTTTGTATAGTTCATCCATGC-3'. The PCR product was then digested with Nhel-BsiWl and inserted into the Nhel-BsiWl sites of YFP-GluAl (obtained from G. Turrigiano, Brandeis University, Waltham, MA). GCaMP2, subcloned into peGFP-N1 vector (Takara Bio Inc.) with Bglll and Notl (Tallini et al., 2006), was obtained from J. Nakai (RIKEN Brain Science Institute, Saitama, Japan).

\section{Imaging solutions}

Standard imaging solution consisted of HBSS (Invitrogen) containing $(\mathrm{mM})$ : 10 Hepes, 2.0 glucose, $1.2 \mathrm{CaCl}_{2}$, and $1.0 \mathrm{MgCl}_{2}$. Synaptic stimulation $\left(\mathrm{OMg}^{2+} / \mathrm{Gly}\right)$ solution contained HBSS supplemented with $(\mathrm{mM})$ : 2 D-glucose, $1.2 \mathrm{CaCl}_{2}$, and 0.2 Glycine (VWR). Depolarizing solution consisted of HBSS supplemented with $(\mathrm{mM}): 40 \mathrm{KCl}, 2.5 \mathrm{CaCl}_{2}$, and $1.0 \mathrm{MgCl}_{2}$. High $\mathrm{Mg}^{2+} /$ low $\mathrm{Ca}^{2+}$ solution consisted of $\mathrm{HBSS}$, supplemented with $(\mathrm{mM})$ : $0.6 \mathrm{CaCl}_{2}$ and $5 \mathrm{MgCl}_{2}$. For the glutamate stimulation (Glu/Gly), $100 \mu \mathrm{M}$ glutamate and $10 \mu \mathrm{M}$ glycine were added to the standard imaging solution. The osmolality of all solutions was adjusted to $260 \mathrm{mOsm} / \mathrm{kg}$ and $\mathrm{pH}$ was adjusted to 7.3. TTX was from Alomone laboratories; AP5, KN92, KN93, and ionomycin were from EMD Millipore; CPA was from Tocris Bioscience; and bicuculline was from Sigma-Aldrich.

\section{Time-lapse imaging}

Time-lapse imaging of mGFP-CaMKII translocation was performed at room temperature essentially as described previously (Bayer et al., 2006) using an upright microscope (Axioskop FS2; Carl Zeiss) with a $63 \times 0.95 \mathrm{NA}$ Achroplan water immersion objective (Carl Zeiss), equipped with a multivalve perfusion system (PTR-2000; ALA Scientific Instruments). For dualcolor imaging, we used an EGFP/dsRed filter set (Chroma Technology Corp.) and sequentially excited mGFP or GCaMP2 (485-nm $25 \times$ filter) and mCherry (565-nm 25x filter) with a Lambda DG-4 system equipped with a 175-W Xenon lamp (Sutter Instrument). GCaMP2 images were acquired every 2-3.5 s with an EMCCD camera (QuantEM:512SC; Photometrics). Except for SEP-GluA1, other live images were acquired every 10-30 s using a CCD camera (CoolSnap-HQ; Photometrics). For CaMKII translocation to microtubules of the different mutants, we acquired a 5 -image $z$-stack at each time point to maximize the detection of fibers across the cell. Time-lapse dual imaging of SEP-GluAl and mCherry-CaMKII was performed at $35^{\circ} \mathrm{C}$ on a microscope (Axiovert 200M; Carl Zeiss) with a $63 \times 1.4$ NA oil immersion objective (Carl Zeiss), equipped with a temperature-controlled perfusion chamber (Warner Instruments) and a multivalve perfusion system (ALA Scientific Instruments). Each minute a z-stack of 7-10 images (0.5- $\mu \mathrm{m}$ step, 50-100-ms integration time per frame) was acquired. At the beginning of an imaging session, while searching for a transfected neuron under the microscope, the cells were bathed in high $\mathrm{Mg}^{2+} /$ low $\mathrm{Ca}^{2+}$ solution, which blocks most of neuronal activity, to prevent spontaneous synaptic translocation of the enzyme. For $0 \mathrm{Mg}^{2+} /$ Gly stimulations, the imaging sequence was as follows: first $2 \mathrm{~min}$ in high $\mathrm{Mg}^{2+} /$ low $\mathrm{Ca}^{2+}$ solution, next $5 \mathrm{~min}$ in standard solution, next $5 \mathrm{~min}$ in $\mathrm{OMg}^{2+} /$ Gly solution, and remaining time in standard solution. For $\mathrm{KCl}$ stimulations, the imaging sequence was: first $2 \mathrm{~min}$ in high $\mathrm{Mg}^{2+} / \mathrm{low}$ $\mathrm{Ca}^{2+}$ solution, next $1 \mathrm{~min}$ in depolarizing solution, and remaining time in high $\mathrm{Mg}^{2+} /$ low $\mathrm{Ca}^{2+}$ solution. For local stimulation, 100/10 $\mu \mathrm{M}$ glutamate/ glycine were added to a standard solution and inserted into a glass pipette with a resistance of $\sim 7 \mathrm{Mohm}$. Trains of 10-70-ms puffs were applied at $2 \mathrm{~Hz}$ using a digital stimulator (Cygnus Technology) controlling a Picospritzer III (Parker Hannifin Corporation) with a pressure set to $\sim 3$ psi.

\section{Live imaging analysis}

Image analyses and deconvolution were performed in MetaMorph (Molecular Devices) or MatLab (The MathWorks, Inc.). Images were automatically aligned using custom software, and background fluorescence was removed. A cell mask was created by manually setting a threshold and removing out-of-focus regions. Statistical analyses were performed in MatLab. All measurements are presented as mean \pm SEM.

mGFP-CaMKII subdendritic translocation. To help the visual detection of CaMKII subdendritic translocation, we subtracted each image of a series by a maximum projection of the images before the stimulation. Possible regions of CaMKII dendritic accumulation were determined in those treated images as a series of contiguous pixels with higher intensity during the stimulation. The region was subsequently verified by examining the raw images to avoid false positive. A line was traced along the dendritic region for each detected CaMKII hot spot and the sum of dendritic length of all those regions for a neuron was calculated and normalized to the total dendritic length in the field of view. To evaluate if the signal from synapses protruding toward the z-axis could be responsible for the subdendritic translocation observed, we acquired z-stacks of thin optical slices by confocal microscopy. We have not found evidence that superimposed spines could account for the dendritic signal.

Dual $\mathrm{Ca}^{2+}$ (GCaMP2) and mCherry-CaMKIl analysis. Dendritic regions exhibiting CaMKII translocation were detected by analyzing an image at the end of the stimulation. Circular regions of $\sim 1.5-3.5 \mu \mathrm{m}^{2}$ were traced, one at the center of each detected site and another one $\sim 5-15 \mu \mathrm{m}$ away within the dendrite. Average intensity in those regions was measured over time $(F)$ and corrected by the mean average intensity before the stimulation $\left(F_{0}\right)$ to obtain $\triangle F / F_{0}$, for both GCaMP2 and mCherry-CaMKII. Over the course of the imaging, we noticed that GCaMP2 signal slightly decreased, possibly due to bleaching and photo-isomerization (Bengtson et al., 2010).

Analysis of mGFP-CaMKII translocation to microtubules. CaMKII translocation to microtubules was automatically analyzed in MetaMorph in a region that includes the soma and proximal dendrites. Each image of a $z$-stack was first deconvolved and a maximum projection was obtained for each time point. A sliding threshold ranging from the image minimum to maximum values in increments of 2 was applied. To detect above-threshold groups of contiguous pixels that corresponded to fiber-like structures, we used four criteria: pixel length corresponding to 2-100 $\mu \mathrm{m}$, fiber breadth (1 / 4 $\left(P-\sqrt{P^{2}-16 A}\right)$; $P$, perimeter; $A$, area) from $0.2-0.5 \mu \mathrm{m}$, breadth from 0.5-0.7 $\mu \mathrm{m}$, and elliptical form factor (length/breadth) $>3.5$. We summed the intensity of all regions detected for each image and normalized it to the total intensity of the cell. We averaged the ratio obtained for the images before the stimulation (before) and at the end of the stimulation (stim: last $30 \mathrm{~s}$ ) and calculated a fold increase stim/before.

mGFP-CaMKII translocation to postsynaptic site. CaMKII translocation to synapses was automatically analyzed in MetaMorph by measurements of the contrast between synaptic and neighboring extrasynaptic pixels. Each image of a z-stack was analyzed separately. A sliding threshold in increments of 10 ranging from the image minimum to maximum values was applied. To detect above-threshold groups of contiguous pixels that corresponded to synapses, we used two criteria: pixel area corresponding to $0.15-2 \mu \mathrm{m}^{2}$ and shape factor $\left(4 \pi \mathrm{A} / \mathrm{P}^{2} ; \mathrm{P}\right.$, perimeter; $\mathrm{A}$, area) $>0.6$. We obtained a maximum projection for each time point of the background-subtracted cell and of the detected clusters. The "synaptic 
contrast" is [(mean pixel intensity of each object) - (threshold value at which it was detected)] / (mean value of all nonzero pixels of the image), averaged over all objects. When mGFP-CaMKII was cotransfected with Homerlc-DsRed (Fig. 8, Ai), Homer synaptic clusters were detected and tracked over time. For each cluster, a size-adapted square $\mathrm{ROI}$ was determined based on morphological analysis and was drawn on each image, according to the tracked position, to measure the associated average intensity of mGFP-CaMKII. For both types of analyses, we averaged the values obtained for the images before the stimulation (before) and during the stimulation and calculated a fold increase stim/before.

Synaptic insertion of GluA 1-containing AMPARs and correlation with dendritic CaMKII translocation. Analysis for SEP-GluAl was done in MatLab using custom software. Deconvolved images from a z-stack were summed for each time point. Those sum projections were then xy aligned before data analysis. Clusters of SEP-GluAl were detected using local threshold and morphological analysis. Clusters with an intensity at least 3 standard deviations higher than the intensity of a 10-15-pixel box surrounding the cluster, with an area $>0.5 \mu^{2}$ and an eccentricity $<0.95$, were selected. The position of these GluAl clusters, considered as synaptic, was then tracked. For each cluster, the size of a region of interest was determined based on the morphological analysis and square $\mathrm{ROI}$ was drawn on each projection image, according to the tracked position, to measure the average intensity in both fluorescence channels. Each synapse trajectory was verified visually to avoid errors in detection. Synaptic CaMKII translocation was determined by comparing the intensity of mGFP- $\alpha$ CaMKII in those synapses before and during the stimulation. CaMKII synaptic accumulation $(\mathrm{S}+)$ : stim/before $>1$, no CaMKII accumulation $(S-)$ : stim/before $\leq 1$. Clear events of CaMKII dendritic translocation were manually identified and synapses either inside $(D+)$ or outside $(D-)$ of this dendritic region were selected for intensity analysis.

Spine number and relationship with dendritic CaMKII translocation. To count spine number, 15- $\mathrm{\mu m}$ sections of dendrites were analyzed separately, blind of the condition. Also blind of the condition, the presence of dendritic CaMKII translocation was determined visually. Using NeuronStudio (Rodriguez et al., 2008), dendrites were drawn semi-automatically and spines were detected. We measured the number of spines for 3 images before and 3 images 30 min after the stimulation to calculate a fold change (after/before). We also classified the spine morphology using the measurements done by NeuronStudio and measured the difference in spine number by category, after versus before the stimulation. Thin spines: head diameter $<0.5 \mu \mathrm{m}$ and length/head diameter $>2$. Mushroom spine: head diameter $>0.5 \mu \mathrm{m}$, head/neck $>1.1$ and length $>0.5 \mu \mathrm{m}$. Stubby spines: others. Occasionally, complex protrusions were detected as more than one spine; because such structure might represent more than one synaptic site, we counted each of them to reflect spine remodeling.

\section{FRAP measurements}

Neurons transfected with $m$ GFP- $\alpha$ CaMKII, preincubated (or not) with $5 \mu M$ nocodazole (Sigma-Aldrich) for $1 \mathrm{~h}$ in the culture medium, were stimulated as described above with $\mathrm{OMg}^{2+} / \mathrm{Gly}$ or $\mathrm{KCl}$. Photobleaching was performed either before, during, or after the stimulation. Images of dendrites were acquired on a confocal system (LSM 510 Meta-Axioskop FS2 Plus; Carl Zeiss) with a $63 \times 0.95$ NA Achroplan water-immersion objective. Scanning illumination was provided by the $488-\mathrm{nm}$ line of a $30-\mathrm{mW}$ argon ion laser set to $25 \%$ of maximum output power. Transmission was set to $4 \%$ for prebleach and recovery imaging. The optical slice was $7 \mu \mathrm{m}$ to collect the maximum fluorescence coming from the spine, which was detected with a long-pass filter $(505 \mathrm{~nm})$ with pixels of $0.02 \mathrm{\mu m}^{2}$. Circular ROls of the same diameter as the dendrite were drawn on dendritic segments. For the $\mathrm{OMg}^{2+} /$ Gly experiments (Fig. $3 \mathrm{~A}$ ), regions of dendritic CaMKII hot spots were first identified during the stimulation by imaging the entire neuron with a CCD camera. In Fig. $3 \mathrm{~A}$, ROIs were traced not directly at the center of dendritic CaMKIl accumulation to minimize the chance of photobleaching a synaptic site, at the expense of underestimating the maximal binding of the enzyme to the spine base. Image acquisition was initiated at a rate of 5 frames/s before bleaching ROls. After 15 frames, bleaching ROls were exposed to 25 scans at $100 \%$ transmission. Imaging resumed immediately after bleaching for $\sim 35 \mathrm{~s}$. The rate of fluorescence recovery in the ROls was evaluated as follows: the pre-photobleach intensity (lpre-bleach) and the photobleach intensity (Ibleach) were rescaled to 1 and 0 , respectively, using the equation:

$$
I_{\text {norm }}(t)=\frac{I(t)-I_{\text {bleach }}}{I_{\text {pre-bleach }}-I_{\text {bleach }}} .
$$

To correct for photobleaching and focus changes during acquisition, we used a correction factor obtained with reference region intensity $\left(\mathrm{I}_{\text {ref }}\right)$ using the equation:

$$
\text { correction factor }=\frac{I_{\text {ref }}-\text { pre-bleach }}{I_{\text {ref }}} .
$$

To collect information from the normalized intensity, we next fitted the function:

$$
I(t)=A\left(1-\sum_{n} \alpha_{n} e^{-B_{n} t}\right)
$$

in MatLab, using the least-squared fit method. The value of $(n)$ is 2. " $A$ " represents the mobile fraction of proteins, " $B$ " the time constant and " $\alpha$ " the magnitude of the exponential. For the $\mathrm{OMg}^{2+} / \mathrm{Gly}$ experiment (Fig. $3 \mathrm{~A}$ ), we fitted only the first $15 \mathrm{~s}$ after the photobleaching to minimize the impact of new binding of $m$ GFP- $\alpha$ CaMKII (i.e., continued accumulation) post-bleaching.

\section{HEK293 cell transfection, stimulation, and analysis}

Human embryonic kidney 293 (HEK293) cells were grown to 30-40\% on poly-D-lysine-coated Aclar coverslips (12 $\mathrm{mm}$ in diameter) in DME growth media supplemented with 10\% fetal bovine serum and Glutamax-1 (Invitrogen). Cells were transfected using Lipofectamine 2000 as described by the manufacturer and treated 16-24 h later. Cells were stimulated or not in a solution containing $\mathrm{HBSS}$ supplemented with $5 \mathrm{mM} \mathrm{CaCl}$ and $10 \mu \mathrm{M}$ ionomycin $(1: 1,000$ dilution of a methanol stock) for 5 min or a solution containing only HBSS, respectively. ROls were drawn in the cells' cytosolic area (outside of nucleus) to measure average intensity in each channel (CFP-cVim, phospho-Vim, and mGFP-CaMKII).

\section{Immunocytochemistry and colocalization analysis}

Neurons were stimulated for $5 \mathrm{~min}$ with $\mathrm{OMg}^{2+} / \mathrm{Gly}$ solution, for $90 \mathrm{~s}$ with depolarizing solution, or for $60 \mathrm{~s}$ with Glu/Gly solution. Immediately after the stimulation, they were fixed for 10 min in $4 \%$ PFA solution (0.1 M phosphate buffer, $4 \%$ sucrose, and 2 mM EGTA, pH 7.4). To assess microtubule stability, using antibodies against tyrosinated and acetylated tubulin, cells were simultaneously permeabilized and fixed in PHEM buffer $(60 \mathrm{mM}$ Pipes, $25 \mathrm{mM}$ Hepes, $5 \mathrm{mM}$ EGTA, and $1 \mathrm{mM} \mathrm{MgCl}$ ) containing also $0.25 \%$ glutaraldehyde, 3.7\% PFA, 3.7\% sucrose, and $0.1 \%$ Triton X-100 (based on Witte et al., 2008). Neurons were then washed once with PBS and twice with 0.1 M PBS/glycine and were permeabilized for $30 \mathrm{~min}$ in blocking solution (PBS, $2 \%$ normal goat serum, and $0.1 \%$ Triton $X-100)$. The following primary antibodies were diluted in blocking solution and incubated for $2 \mathrm{~h}$ at room temperature: mouse anti- $\alpha \mathrm{CaMKII}$ (clone $\mathrm{CB} \alpha 2$, 1:500; Invitrogen), rabbit anti-active CaMKII phospho-T286 (1:500; Promega), rabbit anti-tubulin (1:200; Sigma-Aldrich), rabbit anti-MAP2 (1:500; EMD Millipore), rabbit anti-Neurofilament $M$ (1:500; EMD Millipore), guinea pig anti-VGlut1 (1:500; EMD Millipore), mouse anti-phospho-Vimentin (Ser82, clone MO82, 1:5,000; MBL International), mouse anti-PSD95 (clone 6G6-1C9, 1:500; Cedarlane), mouse anti- $\beta$ CaMKII (clone $C B \beta 1,1: 500$; Invitrogen), mouse anti-acetylated tubulin (1:500; Sigma-Aldrich), and rat anti-tyrosinated tubulin (1:100; Abcam). After washes in PBS, secondary antibodies (goat anti-mouse Alexa Fluor 488, anti-rabbit 546, anti-rabbit 594, anti-guinea pig 633 [1:1,000]; or anti-rat 546 1:500; Invitrogen) were diluted in blocking solution and incubated for $45 \mathrm{~min}$ at room temperature. Coverslips were mounted in Prolong Gold Antifade mounting media (Invitrogen). Images were acquired on a confocal system (LSM510-META Axioskop FS2 Plus; Carl Zeiss) using a $63 \times 1.4$ NA oil immersion objective and an optical slice of $1-1.5 \mu \mathrm{m}$. Alexa Fluor 488 was excited with an argon laser at $488 \mathrm{~nm}$ and detected through a band-pass filter $(505-530 \mathrm{~nm})$. Alexa Fluor 546 and 594 were excited with a HeNe laser at $543 \mathrm{~nm}$ and detected through a band-pass filter $(565-615 \mathrm{~nm})$ and a long-pass filter $(560 \mathrm{~nm})$, respectively. Alexa Fluor 633 was excited with a $\mathrm{HeNe}$ laser at $633 \mathrm{~nm}$ and detected through a band-pass filter $(650-710 \mathrm{~nm})$. To analyze the colocalization between $\alpha \mathrm{CaMKII}$ and microtubules or neurofilaments, images from 40 neurons per condition were treated in MetaMorph to remove background noise and extract regions of interest. Colocalization was assessed by a correlation analysis using a MatLab script derived from Jaskolski et al. (2005), which was originally based 


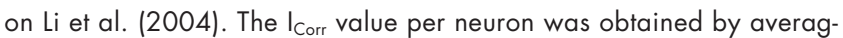
ing the $\mathrm{I}_{\text {Corr }}$ from two ROls selected in proximal dendrites near the soma. The correlation images in Fig. S2 show the normalized mean deviation product (nMDP) pixel map of paired signals.

The nMDP is calculated as follows:

$$
n M D P=\frac{\left(l_{a}-\overline{I_{a}}\right)\left(l_{b}-\overline{l_{b}}\right)}{\left(l_{a \max }-\overline{I_{a}}\right)\left(l_{b \max }-\overline{l_{b}}\right)}
$$

Where la and $I b$ represent the intensity of the pixel in image $A$ and $B$, respectively, $i$ is the mean intensity, and $I_{\max }$ is the maximum pixel value. The nMDP index can vary from -1 to 1 . The $\mathrm{I}_{\text {Corr }}$ index is the ratio of correlated pixels (nMDP > 0) over total pixels. The index varies between 0 and 1 , with 0.5 representing a non-colocalized, random signal.

To analyze the decrease in endogenous CaMKII levels caused by shRNA treatments, we fixed the neurons after live imaging and immunolabeled half of the coverslips for $\alpha$ CaMKII and half for $\beta$ CaMKII because both antibodies are from mouse. We imaged transfected neurons and measured the average intensity of CaMKII immunolabeling in their soma and divided it by the somatic average intensity from other nontransfected neurons in the same field of view.

\section{Online supplemental material}

Fig. S1 shows the patterns of mGFP- $\alpha$ CaMKII dendritic translocation after either Glu/Gly, $\mathrm{OMg}^{2+} / \mathrm{Gly}$, or bicuculline stimulation, or during spontaneous activity of the neurons. Fig. S2 describes the colocalization of endogenous $\alpha$ CaMKII with microtubules after $\mathrm{OMg}^{2+} /$ Gly stimulation or global depolarization with $40 \mathrm{mM} \mathrm{KCl}$. Fig. S3 shows the effect of nocodazole on microtubule destabilization assessed by measuring changes in ratio of acetylated/tyrosinated tubulin. Fig. S4 shows that $\beta$ CaMKII also translocates to local microtubular domains after $\mathrm{OMg}^{2+} / \mathrm{Gly}$ stimulation or to somatodendritic microtubules upon global stimulation with Glu/Gly. Fig. S5 illustrates the morphometric analysis used to quantify microtubular translocation of mGFP-CaMKII during $\mathrm{KCl}$ stimulation as well as the effectiveness of shRNA knockdown of $\alpha$ - and $\beta$ CaMKII in cultured neurons. Video 1 shows a time-lapse sequence of the localized and reversible dendritic translocation of mGFP- $\alpha \mathrm{CaMKII}$ in cultured hippocampal neurons stimulated with $\mathrm{OMg}^{2+} / \mathrm{Gly}$. Video 2 shows a time-lapse sequence of $\mathrm{mGFP}$ $\alpha$ CaMKII dendritic translocation during spontaneous neuronal activity. Video 3 shows a time-lapse sequence demonstrating the repetitive accumulation of $m$ GFP- $\alpha$ CaMKII in subdendritic regions during multiple $\mathrm{OMg}^{2+}$ / Gly stimulations. Video 4 shows time-lapse sequence of $m$ Cherry- $\alpha \mathrm{CaMKII}$ translocation to dendritic regions exhibiting elevated $\mathrm{Ca}^{2+}$ levels, revealed by coincident GCaMP2 imaging. Video 5 shows a time-lapse sequence of $m G F P-\alpha C a M K I I$ translocation to a synapse, followed by the dendritic accumulation of $m$ GFP- $\alpha$ CaMKII onto microtubular-like domains adjacent to that synapse. Video 6 shows a time-lapse sequence of $m$ GFP- $\alpha$ CaMKII decorating somatodendritic microtubules upon neuronal depolarization with $\mathrm{KCl}$. Video 7 shows a time-lapse sequence of $m$ GFP- $\alpha$ CaMKII translocating to a dendritic spine, followed by dendritic accumulation below that spine, and long-lasting change in spine volume, as revealed by simultaneous mCherry time-lapse sequence. Online supplemental material is available at http:// www.jcb.org/cgi/content/full/jcb.201202058/DC1.

We thank Francine Nault for preparing the neuronal cultures and D. Lemelin for recombinant DNA constructions. We thank $\mathrm{H}$. Bito and members of the De Koninck laboratory for helpful comments on the manuscript. We are grateful to Y. Hayashi and S. Lee $(\alpha$ - and $\beta$-CaMKII shRNAs); J. Tsui and H. Schulman (c-Vim); J. Nakai (GCaMP2); G. Miesenbock (Superecliptic pHluorin); and G. Turrigiano (YFP-GluAl) for sharing DNA constructs.

Research was supported by the Canadian Institutes of Health Research (CIHR) and the Natural Science and Engineering Research Council of Canada (NSERC). M. Lemieux was supported by studentships from NSERC, CIHR, and the Fond de la Recherche en Santé du Québec. S. Labrecque was supported by the Neurophysics Training grant. C. Tardif was supported by a studentship from the Fond Québécois de Recherche sur la Nature et les Technologies du Québec and by the Neurophysics Training grant from CIHR. E. Labrie-Dion was supported by a studentship from NSERC. E. LeBel was supported by a studentship from ClHR and the K.M. Hunter foundation. P. De Koninck was a $\mathrm{CIHR}$ Young Investigator Awardee.

Submitted: 10 February 2012

Accepted: 14 August 2012

\section{References}

Augustine, G.J., F. Santamaria, and K. Tanaka. 2003. Local calcium signaling in neurons. Neuron. 40:331-346. http://dx.doi.org/10.1016/S0896-6273 (03)00639-1

Baratier, J., L. Peris, J. Brocard, S. Gory-Fauré, F. Dufour, C. Bosc, A. Fourest-Lieuvin, L. Blanchoin, P. Salin, D. Job, and A. Andrieux. 2006. Phosphorylation of microtubule-associated protein STOP by calmodulin kinase II. J. Biol. Chem. 281:19561-19569. http://dx.doi.org/10.1074/ jbc.M509602200

Baudier, J., and R.D. Cole. 1987. Phosphorylation of tau proteins to a state like that in Alzheimer's brain is catalyzed by a calcium/calmodulindependent kinase and modulated by phospholipids. J. Biol. Chem. 262:17577-17583.

Bayer, K.U., P. De Koninck, A.S. Leonard, J.W. Hell, and H. Schulman. 2001. Interaction with the NMDA receptor locks CaMKII in an active conformation. Nature. 411:801-805. http://dx.doi.org/10.1038/35081080

Bayer, K.U., E. LeBel, G.L. McDonald, H. O'Leary, H. Schulman, and P. De Koninck. 2006. Transition from reversible to persistent binding of CaMKII to postsynaptic sites and NR2B. J. Neurosci. 26:1164-1174. http://dx.doi.org/10.1523/JNEUROSCI.3116-05.2006

Bengtson, C.P., H.E. Freitag, J.M. Weislogel, and H. Bading. 2010. Nuclear calcium sensors reveal that repetition of trains of synaptic stimuli boosts nuclear calcium signaling in CA1 pyramidal neurons. Biophys. J. 99: 4066-4077. http://dx.doi.org/10.1016/j.bpj.2010.10.044

Branco, T., and M. Häusser. 2010. The single dendritic branch as a fundamental functional unit in the nervous system. Curr. Opin. Neurobiol. 20:494-502. http://dx.doi.org/10.1016/j.conb.2010.07.009

Chalifoux, J.R., and A.G. Carter. 2011. Glutamate spillover promotes the generation of NMDA spikes. J. Neurosci. 31:16435-16446. http://dx.doi .org/10.1523/JNEUROSCI.2777-11.2011

Colbran, R.J. 2004. Targeting of calcium/calmodulin-dependent protein kinase II. Biochem. J. 378:1-16. http://dx.doi.org/10.1042/BJ20031547

Conde, C., and A. Cáceres. 2009. Microtubule assembly, organization and dynamics in axons and dendrites. Nat. Rev. Neurosci. 10:319-332. http:// dx.doi.org/10.1038/nrn2631

Correia, S.S., S. Bassani, T.C. Brown, M.F. Lisé, D.S. Backos, A. El-Husseini, M. Passafaro, and J.A. Esteban. 2008. Motor protein-dependent transport of AMPA receptors into spines during long-term potentiation. Nat. Neurosci. 11:457-466. http://dx.doi.org/10.1038/nn2063

Emptage, N., T.V. Bliss, and A. Fine. 1999. Single synaptic events evoke NMDA receptor-mediated release of calcium from internal stores in hippocampal dendritic spines. Neuron. 22:115-124. http://dx.doi.org/10.1016/ S0896-6273(00)80683-2

Fink, C.C., K.U. Bayer, J.W. Myers, J.E. Ferrell Jr., H. Schulman, and T. Meyer. 2003. Selective regulation of neurite extension and synapse formation by the beta but not the alpha isoform of CaMKII. Neuron. 39:283-297. http://dx.doi.org/10.1016/S0896-6273(03)00428-8

Frey, U., and R.G. Morris. 1998. Synaptic tagging: implications for late maintenance of hippocampal long-term potentiation. Trends Neurosci. 21:181-188. http://dx.doi.org/10.1016/S0166-2236(97)01189-2

Govindarajan, A., R.J. Kelleher, and S. Tonegawa. 2006. A clustered plasticity model of long-term memory engrams. Nat. Rev. Neurosci. 7:575-583. http://dx.doi.org/10.1038/nrn1937

Govindarajan, A., I. Israely, S.Y. Huang, and S. Tonegawa. 2011. The dendritic branch is the preferred integrative unit for protein synthesis-dependent LTP. Neuron. 69:132-146. http://dx.doi.org/10.1016/j.neuron.2010.12.008

Guillaud, L., R. Wong, and N. Hirokawa. 2008. Disruption of KIF17-Mint1 interaction by CaMKII-dependent phosphorylation: a molecular model of kinesin-cargo release. Nat. Cell Biol. 10:19-29. http://dx.doi.org/10.1038/ ncb1665

Hanson, P.I., and H. Schulman. 1992. Neuronal Ca2+/calmodulin-dependent protein kinases. Annu. Rev. Biochem. 61:559-601. http://dx.doi.org/10.1146/ annurev.bi.61.070192.003015

Hanson, P.I., T. Meyer, L. Stryer, and H. Schulman. 1994. Dual role of calmodulin in autophosphorylation of multifunctional CaM kinase may underlie decoding of calcium signals. Neuron. 12:943-956. http://dx.doi.org/ 10.1016/0896-6273(94)90306-9

Hardie, J., and N. Spruston. 2009. Synaptic depolarization is more effective than back-propagating action potentials during induction of associative long-term potentiation in hippocampal pyramidal neurons. J. Neurosci. 29:3233-3241. http://dx.doi.org/10.1523/JNEUROSCI.6000-08.2009

Harvey, C.D., and K. Svoboda. 2007. Locally dynamic synaptic learning rules in pyramidal neuron dendrites. Nature. 450:1195-1200. http://dx.doi.org/ 10.1038 /nature06416

Hayashi, Y., S.H. Shi, J.A. Esteban, A. Piccini, J.C. Poncer, and R. Malinow. 2000. Driving AMPA receptors into synapses by LTP and CaMKII: 
requirement for GluR1 and PDZ domain interaction. Science. 287:22622267. http://dx.doi.org/10.1126/science.287.5461.2262

Higley, M.J., and B.L. Sabatini. 2008. Calcium signaling in dendrites and spines: practical and functional considerations. Neuron. 59:902-913. http:// dx.doi.org/10.1016/j.neuron.2008.08.020

Hudmon, A., E. Lebel, H. Roy, A. Sik, H. Schulman, M.N. Waxham, and P. De Koninck. 2005. A mechanism for Ca2+/calmodulin-dependent protein kinase II clustering at synaptic and nonsynaptic sites based on self-association. J. Neurosci. 25:6971-6983. http://dx.doi.org/10.1523/ JNEUROSCI.4698-04.2005

Jaskolski, F., C. Mulle, and O.J. Manzoni. 2005. An automated method to quantify and visualize colocalized fluorescent signals. J. Neurosci. Methods. 146:42-49. http://dx.doi.org/10.1016/j.jneumeth.2005.01.012

Jourdain, P., K. Fukunaga, and D. Muller. 2003. Calcium/calmodulin-dependent protein kinase II contributes to activity-dependent filopodia growth and spine formation. J. Neurosci. 23:10645-10649.

Karcher, R.L., J.T. Roland, F. Zappacosta, M.J. Huddleston, R.S. Annan, S.A. Carr, and V.I. Gelfand. 2001. Cell cycle regulation of myosin-V by calcium/ calmodulin-dependent protein kinase II. Science. 293:1317-1320. http:// dx.doi.org/10.1126/science.1061086

Kleindienst, T., J. Winnubst, C. Roth-Alpermann, T. Bonhoeffer, and C. Lohmann. 2011. Activity-dependent clustering of functional synaptic inputs on developing hippocampal dendrites. Neuron. 72:1012-1024. http://dx.doi.org/10.1016/j.neuron.2011.10.015

Korkotian, E., and M. Segal. 2007. Morphological constraints on calcium dependent glutamate receptor trafficking into individual dendritic spine. Cell Calcium. 42:41-57. http://dx.doi.org/10.1016/j.ceca.2006.11.006

Lee, S.J., Y. Escobedo-Lozoya, E.M. Szatmari, and R. Yasuda. 2009. Activation of CaMKII in single dendritic spines during long-term potentiation. Nature. 458:299-304. http://dx.doi.org/10.1038/nature07842

Li, Q., A. Lau, T.J. Morris, L. Guo, C.B. Fordyce, and E.F. Stanley. 2004. A syntaxin 1, Galpha(o), and N-type calcium channel complex at a presynaptic nerve terminal: analysis by quantitative immunocolocalization. J. Neurosci. 24:4070-4081. http://dx.doi.org/10.1523/JNEUROSCI.0346-04.2004

Lisman, J., R. Yasuda, and S. Raghavachari. 2012. Mechanisms of CaMKII action in long-term potentiation. Nat. Rev. Neurosci. 13:169-182.

Lohmann, C., and T. Bonhoeffer. 2008. A role for local calcium signaling in rapid synaptic partner selection by dendritic filopodia. Neuron. 59:253-260. http://dx.doi.org/10.1016/j.neuron.2008.05.025

Lu, W., H. Man, W. Ju, W.S. Trimble, J.F. MacDonald, and Y.T. Wang. 2001. Activation of synaptic NMDA receptors induces membrane insertion of new AMPA receptors and LTP in cultured hippocampal neurons. Neuron. 29:243-254. http://dx.doi.org/10.1016/S0896-6273(01)00194-5

Maas, C., D. Belgardt, H.K. Lee, F.F. Heisler, C. Lappe-Siefke, M.M. Magiera, J. van Dijk, T.J. Hausrat, C. Janke, and M. Kneussel. 2009. Synaptic activation modifies microtubules underlying transport of postsynaptic cargo. Proc. Natl. Acad. Sci. USA. 106:8731-8736. http://dx.doi.org/ 10.1073/pnas.0812391106

Makino, H., and R. Malinow. 2009. AMPA receptor incorporation into synapses during LTP: the role of lateral movement and exocytosis. Neuron. 64:381-390. http://dx.doi.org/10.1016/j.neuron.2009.08.035

Maletic-Savatic, M., T. Koothan, and R. Malinow. 1998. Calcium-evoked dendritic exocytosis in cultured hippocampal neurons. Part II: mediation by calcium/calmodulin-dependent protein kinase II. J. Neurosci. 18:6814-6821.

Marsden, K.C., A. Shemesh, K.U. Bayer, and R.C. Carroll. 2010. Selective translocation of $\mathrm{Ca} 2+/$ calmodulin protein kinase IIalpha (CaMKIIalpha) to inhibitory synapses. Proc. Natl. Acad. Sci. USA. 107:20559-20564. http://dx.doi.org/10.1073/pnas.1010346107

Matsuzaki, M., N. Honkura, G.C. Ellis-Davies, and H. Kasai. 2004. Structural basis of long-term potentiation in single dendritic spines. Nature. 429:761-766. http://dx.doi.org/10.1038/nature02617

Nault, F., and P. De Koninck. 2010. Dissociated hippocampal neurons. In Protocols in Neural Cell Cultures. Springer Protocols Handbooks. 137-160.

Opazo, P., S. Labrecque, C.M. Tigaret, A. Frouin, P.W. Wiseman, P. De Koninck, and D. Choquet. 2010. CaMKII triggers the diffusional trapping of surface AMPARs through phosphorylation of stargazin. Neuron. 67:239252. http://dx.doi.org/10.1016/j.neuron.2010.06.007

Otmakhov, N., J.H. Tao-Cheng, S. Carpenter, B. Asrican, A. Dosemeci, T.S. Reese, and J. Lisman. 2004. Persistent accumulation of calcium/calmodulindependent protein kinase II in dendritic spines after induction of NMDA receptor-dependent chemical long-term potentiation. J. Neurosci. 24:93249331. http://dx.doi.org/10.1523/JNEUROSCI.2350-04.2004

Park, M., J.M. Salgado, L. Ostroff, T.D. Helton, C.G. Robinson, K.M. Harris, and M.D. Ehlers. 2006. Plasticity-induced growth of dendritic spines by exocytic trafficking from recycling endosomes. Neuron. 52:817-830. http://dx.doi.org/10.1016/j.neuron.2006.09.040
Patterson, M.A., E.M. Szatmari, and R. Yasuda. 2010. AMPA receptors are exocytosed in stimulated spines and adjacent dendrites in a Ras-ERK-dependent manner during long-term potentiation. Proc. Natl. Acad. Sci. USA. 107: 15951-15956. http://dx.doi.org/10.1073/pnas.0913875107

Pi, H.J., N. Otmakhov, F. El Gaamouch, D. Lemelin, P. De Koninck, and J. Lisman. 2010. CaMKII control of spine size and synaptic strength: role of phosphorylation states and nonenzymatic action. Proc. Natl. Acad. Sci. USA. 107:14437-14442. http://dx.doi.org/10.1073/pnas .1009268107

Redondo, R.L., and R.G. Morris. 2011. Making memories last: the synaptic tagging and capture hypothesis. Nat. Rev. Neurosci. 12:17-30. http:// dx.doi.org/10.1038/nrn2963

Rich, R.C., and H. Schulman. 1998. Substrate-directed function of calmodulin in autophosphorylation of Ca2+/calmodulin-dependent protein kinase II. J.Biol. Chem. 273:28424-28429.http://dx.doi.org/10.1074/jbc.273.43 .28424

Robison, A.J., M.A. Bass, Y. Jiao, L.B. MacMillan, L.C. Carmody, R.K. Bartlett, and R.J. Colbran. 2005. Multivalent interactions of calcium/calmodulindependent protein kinase II with the postsynaptic density proteins NR2B, densin-180, and alpha-actinin-2. J. Biol. Chem. 280:35329-35336. http:// dx.doi.org/10.1074/jbc.M502191200

Rodriguez, A., D.B. Ehlenberger, D.L. Dickstein, P.R. Hof, and S.L. Wearne. 2008. Automated three-dimensional detection and shape classification of dendritic spines from fluorescence microscopy images. PLoS One. 3:e1997.

Rose, J., S.X. Jin, and A.M. Craig. 2009. Heterosynaptic molecular dynamics: locally induced propagating synaptic accumulation of CaM kinase II. Neuron. 61:351-358. http://dx.doi.org/10.1016/j.neuron.2008.12.030

Schiller, J., Y. Schiller, and D.E. Clapham. 1998. NMDA receptors amplify calcium influx into dendritic spines during associative pre- and postsynaptic activation. Nat. Neurosci. 1:114-118. http://dx.doi.org/10.1038/693

Schiller, J., G. Major, H.J. Koester, and Y. Schiller. 2000. NMDA spikes in basal dendrites of cortical pyramidal neurons. Nature. 404:285-289. http:// dx.doi.org/10.1038/35005094

Schulman, H. 1984. Phosphorylation of microtubule-associated proteins by a Ca2+/calmodulin-dependent protein kinase. J. Cell Biol. 99:11-19. http:// dx.doi.org/10.1083/jcb.99.1.11

Shen, K., and T. Meyer. 1999. Dynamic control of CaMKII translocation and localization in hippocampal neurons by NMDA receptor stimulation. Science. 284:162-166. http://dx.doi.org/10.1126/science.284.5411.162

Sobczyk, A., V. Scheuss, and K. Svoboda. 2005. NMDA receptor subunit-dependent $[\mathrm{Ca} 2+]$ signaling in individual hippocampal dendritic spines. J. Neurosci. 25:6037-6046. http://dx.doi.org/10.1523/JNEUROSCI.1221-05.2005

Steward, O., and S. Halpain. 1999. Lamina-specific synaptic activation causes domain-specific alterations in dendritic immunostaining for MAP2 and CAM kinase II. J. Neurosci. 19:7834-7845.

Sutton, M.A., and E.M. Schuman. 2006. Dendritic protein synthesis, synaptic plasticity, and memory. Cell. 127:49-58. http://dx.doi.org/10.1016/ j.cell.2006.09.014

Takahashi, N., K. Kitamura, N. Matsuo, M. Mayford, M. Kano, N. Matsuki, and Y. Ikegaya. 2012. Locally synchronized synaptic inputs. Science. 335:353-356. http://dx.doi.org/10.1126/science.1210362

Tallini, Y.N., M. Ohkura, B.R. Choi, G. Ji, K. Imoto, R. Doran, J. Lee, P. Plan, J. Wilson, H.B. Xin, et al. 2006. Imaging cellular signals in the heart in vivo: Cardiac expression of the high-signal $\mathrm{Ca} 2+$ indicator GCaMP2. Proc. Natl. Acad. Sci. USA. 103:4753-4758. http://dx.doi .org/10.1073/pnas.0509378103

Toni, N., P.A. Buchs, I. Nikonenko, C.R. Bron, and D. Muller. 1999. LTP promotes formation of multiple spine synapses between a single axon terminal and a dendrite. Nature. 402:421-425. http://dx.doi.org/10.1038/46574

Vallano, M.L., J.R. Goldenring, R.S. Lasher, and R.J. Delorenzo. 1986. Association of calcium/calmodulin-dependent kinase with cytoskeletal preparations: phosphorylation of tubulin, neurofilament, and microtubule-associated proteins. Ann. N.Y.Acad. Sci. 466:357-374. http://dx.doi .org/10.1111/j.1749-6632.1986.tb38406.x

Wang, Z., J.G. Edwards, N. Riley, D.W. Provance Jr., R. Karcher, X.D. Li, I.G. Davison, M. Ikebe, J.A. Mercer, J.A. Kauer, and M.D. Ehlers. 2008. Myosin $\mathrm{Vb}$ mobilizes recycling endosomes and AMPA receptors for postsynaptic plasticity. Cell. 135:535-548. http://dx.doi.org/10.1016/ j.cell.2008.09.057

Witte, H., D. Neukirchen, and F. Bradke. 2008. Microtubule stabilization specifies initial neuronal polarization. J. Cell Biol. 180:619-632. http:// dx.doi.org/10.1083/jcb.200707042

Xie, Z., D.P. Srivastava, H. Photowala, L. Kai, M.E. Cahill, K.M. Woolfrey, C.Y. Shum, D.J. Surmeier, and P. Penzes. 2007. Kalirin-7 controls activitydependent structural and functional plasticity of dendritic spines. Neuron. 56:640-656. http://dx.doi.org/10.1016/j.neuron.2007.10.005 
Yamagata, Y., S. Kobayashi, T. Umeda, A. Inoue, H. Sakagami, M. Fukaya, M. Watanabe, N. Hatanaka, M. Totsuka, T. Yagi, et al. 2009. Kinasedead knock-in mouse reveals an essential role of kinase activity of $\mathrm{Ca} 2+/$ calmodulin-dependent protein kinase IIalpha in dendritic spine enlargement, long-term potentiation, and learning. J. Neurosci. 29:76077618. http://dx.doi.org/10.1523/JNEUROSCI.0707-09.2009

Yang, E., and H. Schulman. 1999. Structural examination of autoregulation of multifunctional calcium/calmodulin-dependent protein kinase II. J. Biol. Chem. 274:26199-26208. http://dx.doi.org/10.1074/jbc.274.37 .26199

Zacharias, D.A., J.D. Violin, A.C. Newton, and R.Y. Tsien. 2002. Partitioning of lipid-modified monomeric GFPs into membrane microdomains of live cells. Science. 296:913-916. http://dx.doi.org/10.1126/science.1068539 